\title{
A Guide to Localized Frames and Applications to Galerkin-like Representations of Operators
}

\author{
Peter Balazs and Karlheinz Gröchenig
}

\begin{abstract}
This chapter offers a detailed survey on intrinsically localized frames and the corresponding matrix representation of operators. We re-investigate the properties of localized frames and the associated Banach spaces in full detail. We investigate the representation of operators using localized frames in a so-called Galerkintype scheme. We show how the boundedness and the invertibility of matrices and operators are linked and give some sufficient and necessary conditions for the boundedness of operators between the associated Banach spaces.
\end{abstract}

\section{Introduction}

Localized frames are "good" frames. More precisely, the concept of localized frames was introduced in [50] in an attempt to understand which properties render a frame useful. Whereas an abstract frame can be viewed as a flexible coordinate system for a Hilbert space - and only for one Hilbert space! - localized frames go beyond Hilbert spaces and yield a description and characterization of a whole class of associated Banach spaces. Indeed, the success of structured frames, such as Gabor frames [36], wavelet frames [30], or frames of translates [11], is built on their capacity to describe modulation spaces (Gabor), Besov-Triebel-Lizorkin spaces (wavelet), and their use in sampling theory (translates).

Gabor frames are used for the description and extraction of time-frequency features of a signal. It would be a waste of possibilities to use them to merely determine the $L^{2}$-membership of a given function. Likewise wavelets are used to detect edges

Peter Balazs

Acoustics Research Institute, Austrian Academy of Sciences, Wohllebengasse 12-14, Vienna, 1040, Austria e-mail: peter.balazs@oeaw.ac.at

Karlheinz Gröchenig

Faculty of Mathematics, University of Vienna, Oskar-Morgenstern-Platz 1, Vienna, 1090, Austria e-mail: karlheinz.groechenig@univie.ac.at 
in an image or compress an image, and not just for the expansion of a function in $L^{2}$. In these applications one does not use arbitrary Gabor frames or wavelet frames, but the window and the wavelet are usually carefully designed so as to have some desired time-frequency concentration or a small support and vanishing moments. Thus in such applications the frames come with an additional property, namely some form of localization.

The general theory of localized frames began as an attempt to formulate an abstract concept of localization that can explain the success of certain structured frames in applications [50]. Roughly speaking, a frame $\Psi=\left\{\psi_{k}: k \in K\right\}$ is called localized, if its Gramian matrix $G$ with entries $G_{k, l}=\left\langle\psi_{l}, \psi_{k}\right\rangle_{k, l \in K}$ possesses enough off-diagonal decay. In the further developments of the concept powerful methods of Banach algebra theory were used, and nowadays, and also in this survey, we call a frame localized, if its Gramian belongs to a solid, inverse-closed Banach algebra of matrices [38].

Localized frames possess many properties that are not available for general frames.

(i) To every localized frame can be associated a class of Banach spaces, the socalled coorbit spaces [34, 35]. Roughly speaking, the coorbit space $\mathscr{H}_{w}^{p}$ contains all elements $f$ such that the sequence $\left\langle f, \psi_{k}\right\rangle_{k}$ belongs to the weighted $\ell^{p}$-space. For the standard structured frames one obtains well-known and classical families of functions spaces, namely, the modulation spaces are associated to Gabor frames [47], and the Besov spaces are associated to wavelet frames [69, 78]. In this chapter we will explain the construction of the coorbit spaces and derive many of their properties.

(ii) Localized frames possess nice dual frames. Technically speaking, the canonical dual frame possesses the same localization. In fact, this is a fundamental statement about localized frames, and the method of proof (matrix algebras, spectral invariance) has motivated intensive research in Banach algebra theory [55, 57, 54, 53].

(iii) Elements in coorbit spaces associated to a localized frame possess good approximation properties [21]. In fact, the results on nonlinear $N$-term approximations and on the fast convergence of the iterative frame algorithms are based fundamentally on the localization property and do not hold for arbitrary frames.

(iv) Localized frames often possess a characterization "without inequalities" [52, [56, 31]. These results have lead to strong results about Gabor frames that have been out of reach with conventional methods.

(v) Every localized frame is a finite union of Riesz sequences [49]. This is a special case of the Feichtinger conjecture and was proved early on with an easy proof, whereas the recent full proof of the Feichtinger conjecture is a monumental result in functional analysis and occupies a completely different mathematical universe [68].

(vi) General frames may be used to describe and discretize operators and operator equations, and thus have led to an important line of frame research in numerical analysis. In the so-called Galerkin approach an operator $O$ is discretized with respect to a frame by associating a matrix $M$, with $M_{k, l}=\left\langle O \psi_{k}, \phi_{l}\right\rangle$ with respect to given frames $\Psi, \Phi$. The standard discretization uses bases, but recently also frames 
have been used [5]. The Galerkin approach works particularly well when the corresponding matrix is sparse. The most famous example is the sparsification of singular integral operators by means of wavelet frames (or bases) [42, 70]. This work has led to many adaptive methods [60, 75, 28]. In this regard the time-frequency analysis of pseudodifferential operators by means of Gabor frames is a particularly successful example of the application of localized frames: certain symbol classes containing the Hörmander class $S_{0,0}^{0}$ can be completely characterized by the off-diagonal decay of the associated matrix [57]. Subsequently Gabor frames were pivotal for the sparsification of Fourier integral operators and certain Schrödinger propagators in the work of the Torino group [20, 22, 23, 24] On a more abstract level, localized frames have been heavily used in the adaptive frame methods for the solution of operator equations in [27, 26].

This chapter will offer a survey of localized frames. Of the many possible topics we will focus on the theory of the associated coorbit spaces and on the Galerkin discretization of operators with respect to localized frames. We will mainly explain the abstract theory and focus on the formalism of localized frames. These aspects have not received as much attention as other topics and justify a more thorough treatment. Several results may even claim some novelty, for instance, the inclusion relations of coorbit spaces and the explicit relation between the mapping properties of an operator and of its corresponding matrix seem to be new.

Although the topic of localized frames is eminently applied, we will choose a formalistic point of view and develop and explain the formalism of localized frames, their coorbit spaces, and the Galerkin discretization.

The motivation for this formal approach, and for this entire chapter, comes from different readings of the original sources [50, 38] and many ensuing discussions between the authors. One of us (K. G.) claimed that "this is folklore and known", while the other (P. B.) would point out - and rightly so - that the results he needed and wanted to understand in detail were not formulated in the publications. P. B. strongly argued that he needed a general abstract formalism in order move on to the real applications in acoustic applications as e.g. in [72]. The result of our discussions is this summary of localized frames with its emphasis on the formalism. We hope that this point of view will also benefit other readers and users of localized frames.

This chapter is organized as follows. In Section 2 we collect some preliminary definitions and notation and then introduce the concept of localization frames. Section 3 is devoted to the study of the associated coorbit spaces and the canonical operators associated to a frame. In Section 4 we describe the Galerkin approach and discuss the formalism of matrix representations of operators with respect to localized frames. We end with a short outlook in Section 5 


\section{Preliminaries and Notation}

For a standard reference to functional analysis and operator theory refer e.g. to [18]. We denote by $\mathfrak{B}(X)$ the Banach algebra of bounded operators on the normed space $X$. We will write $\|T\|_{X \rightarrow Y}$ for the operator norm of a bounded operator $T: X \rightarrow Y$, or just $\|T\|$, if the spaces are clear. We will use the same notation for the inner product of a Hilbert space $\langle., .\rangle_{\mathscr{H}}$ and for the duality relation of two dual sets $B, B^{\prime},\langle., .\rangle_{B, B^{\prime}}$. If there is no chance of confusion, we will just use the notation $\langle.,$.$\rangle for that.$

Let $A \in \mathfrak{B}\left(\mathscr{H}_{1}, \mathscr{H}_{2}\right)$ with closed range. Then there exists a unique bounded operator $A^{\dagger}: \mathscr{H}_{2} \rightarrow \mathscr{H}_{1}$ for which $A A^{\dagger} f=f, \forall f \in \operatorname{ran}(A)$ and $\operatorname{ker}\left(A^{\dagger}\right)=(\operatorname{ran}(A))^{\perp}$. This $A^{\dagger}$ is called the (Moore-Penrose) pseudoinverse of $A$. See e.g. [15].

\subsection{Sequence spaces}

We use the canonical notation of sequence spaces $\ell^{p}=\ell^{p}(K)$ consisting of sequences on a countable and separable index set $K \subseteq \mathbb{R}^{d}$. By an abuse of notation, but for greater consistency, we define $\ell^{0}$ as those sequences for which $\lim _{k \rightarrow \infty} c_{k}=0$. (Usually this space is denoted by $c_{0}$.) We denote the set of sequence with only finitely many non-zero entries by $\ell^{00}$ (usually denoted by $c_{00}$ ).

A weight is a (strictly) positive sequence $w=\left(w_{k}\right)_{k \in K}, w_{k}>0$. Then we define the weighted space $\ell_{w}^{p}$ by $c \in \ell_{w}^{p} \Longleftrightarrow w \cdot c \in \ell^{p}$ with norm $\|c\|_{\ell_{w}^{p}}=\|c \cdot w\|_{\ell^{p}}$. So for the weighted sequence spaces and $1<p \leq 2$ and $2 \leq q<\infty$ we get

$$
\ell^{00} \subseteq \underbrace{\ell_{w}^{1} \subseteq \ell_{w}^{p} \subseteq \ell_{w}^{2} \subseteq \ell_{w}^{q} \subseteq \ell_{w}^{0}}_{(*)} \subseteq \ell_{w}^{\infty}
$$

where the middle $(*)$ is a chain of dense Banach spaces. $\ell^{00}$ is dense in all $\ell_{w}^{p}$ for $1 \leq p<\infty$ and $p=0$, and weak-* dense in $\ell_{w}^{\infty}$. Clearly $\ell_{w}^{0}=\overline{\ell^{00}}\|\cdot\|_{\ell_{w}}$.

For $1 \leq p<\infty$ and $1=\frac{1}{p}+\frac{1}{q}$ we know that $\left(\ell_{w}^{p}\right)^{\prime} \cong \ell_{1 / w}^{q}$ with the duality relation

$$
\left\langle c_{k}, d_{k}\right\rangle_{\ell_{w}^{p}, \ell_{1 / w}^{q}}=\left\langle w_{k} c_{k}, \frac{1}{w_{k}} d_{k}\right\rangle_{l^{p}, l^{q}}=\sum_{k} c_{k} \overline{d_{k}} .
$$

For $p=\infty$ this is only true in the Köthe dual sense [62]. We also have $\left(l_{w}^{0}\right)^{\prime} \cong \ell_{1 / w}^{1}$. 


\subsection{Frames}

A sequence $\Psi=\left(\psi_{k}\right)_{k \in K}$ in a separable Hilbert space $\mathscr{H}$ is a frame for $\mathscr{H}$, if there exist positive constants $A$ and $B$ (called lower and upper frame bound, respectively) that satisfy

$$
A\|f\|^{2} \leq \sum_{k \in K}\left|\left\langle f, \psi_{k}\right\rangle\right|^{2} \leq B\|f\|^{2} \forall f \in \mathscr{H} .
$$

A frame where the two bounds can be chosen to be equal, i.e., $A=B$, is called tight. In the following we will denote by $\Psi=\left(\psi_{k}\right)$ and $\Phi=\left(\phi_{k}\right)$ the corresponding sequences in $\mathscr{H}$.

By $C_{\Psi}: \mathscr{H} \rightarrow \ell^{2}$ we denote the analysis operator defined by $\left(C_{\Psi} f\right)_{k}=\left\langle f, \psi_{k}\right\rangle$. The adjoint of $C_{\Psi}$ is the synthesis operator $D_{\Psi}\left(c_{k}\right)=\sum_{k} c_{k} \psi_{k}$. The frame operator $S_{\Psi}=D_{\Psi} C_{\Psi}$ can be written as $S_{\Psi} f=\sum_{k}\left\langle f, \psi_{k}\right\rangle \psi_{k}$, it is positive and invertible 1 . By using the canonical dual frame $\left(\tilde{\psi}_{k}\right), \tilde{\psi}_{k}=S^{-1} \psi_{k}$ for all $k$, we obtain the reconstruction formula

$$
f=\sum_{k}\left\langle f, \psi_{k}\right\rangle \tilde{\psi}_{k}=\sum_{k}\left\langle f, \tilde{\psi}_{k}\right\rangle \psi_{k} \quad \text { for all } f \in \mathscr{H} .
$$

Any sequence $\Psi_{d}=\left(\psi_{k}^{d}\right)$ for which $C_{\Psi^{d}}$ is bounded on $\mathscr{H}$ and where such a reconstruction holds is called a dual frame.

The Gram matrix $G_{\Psi}$ is defined by $\left(G_{\Psi}\right)_{k, l}=\left\langle\psi_{l}, \psi_{k}\right\rangle$. This matrix defines an operator on $\ell^{2}$ by matrix multiplication, corresponding to $G_{\Psi}=C_{\Psi} D_{\Psi}$. Similarily we can define the cross-Gram matrix $\left(G_{\Psi, \Phi}\right)_{k, l}=\left\langle\phi_{l}, \psi_{k}\right\rangle$ for two frames $\Phi$ and $\Psi$. Clearly

$$
G \Psi, \Phi c=\sum_{l}(G \Psi, \Phi)_{k, l} c_{l}=\left\langle\sum_{l} c_{l} \phi_{l}, \psi_{k}\right\rangle=C_{\Psi} D_{\Phi} c .
$$

If, for the sequence $\Psi$, there exist constants $A, B>0$ such that the inequalities

$$
A\|c\|_{2}^{2} \leq\left\|\sum_{k \in K} c_{k} \psi_{k}\right\|_{\mathscr{H}}^{2} \leq B\|c\|_{2}^{2}
$$

are fulfilled, $\Psi$ is called a Riesz sequence. If $\Psi$ is complete, it is called a Riesz basis.

\subsubsection{Banach frames}

The concept of frames can be extended to Banach spaces [46, 17, 12]:

Let $X$ be a Banach space and $X_{d}$ be a Banach space of scalar sequences. A sequence $\left(\psi_{k}\right)$ in the dual $X^{\prime}$ is called a $X_{d}$-frame for the Banach space $X$ if there exist constants $A, B>0$ such that

\footnotetext{
${ }^{1}$ Note that those 'frame-related' operators can be defined as possibly unbounded operators for any sequence [8].
} 


$$
A\|f\|_{X} \leq\left\|\left\langle f, \psi_{k}\right\rangle_{k \in K}\right\|_{X_{d}} \leq B\|f\|_{X} \quad \text { for all } \quad f \in X .
$$

An $X_{d}$-frame is called a Banach frame with respect to a sequence space $X_{d}$, if there exists a bounded reconstruction operator $R: X_{d} \rightarrow X$, such that $R\left(\psi_{k}(f)\right)=f$ for all $f \in X$. If $X_{d}=\ell^{p}$ for $1 \leq p \leq \infty$, we speak of $p$-frames, respectively $p$ Banach frames. The distinction between $X_{d}$-frames and Banach frames will disappear for localized frames. The norm equivalence (4) already implies the existence of a reconstruction operator for $X$, in this setting.

\subsubsection{Gelfand triples}

Let $X$ be a Banach space and $\mathscr{H}$ a Hilbert space. Then the triple $\left(X, \mathscr{H}, X^{\prime}\right)$ is called a Banach Gelfand triple, if $X \subseteq \mathscr{H} \subseteq X^{\prime}$, where $X$ is dense in $\mathscr{H}$, and $\mathscr{H}$ is $w^{*}$-dense in $X^{\prime}$. The prototype of such a triple is $\left(\ell^{1}, \ell^{2}, \ell^{\infty}\right)$.

A frame for $\mathscr{H}$ is called a Gelfand frame [27] for this triple if there exists a Gelfand triple of sequence spaces $\left(X_{d}, \ell^{2}, X_{d}^{\prime}\right)$, such that the synthesis operator $D \Psi$ : $X_{d} \rightarrow X$ and the analysis operator $C_{\tilde{\Psi}}: X \rightarrow X_{d}$ are bounded.

Now for a Gelfand frame $\Psi$ for the Gelfand triple $\left(X, \mathscr{H}, X^{\prime}\right)$ with the sequence spaces $\left(\ell^{1}, \ell^{2}, \ell^{\infty}\right)$, we define the coorbit space $\mathscr{C} o\left(\ell^{p}, \Psi\right)=\left\{f \in X^{\prime}: C_{\Psi} f \in \ell^{p}\right\}$. Similarly, one could define the orbit spaces $\mathscr{O}\left(\ell^{p}, \Psi\right)=\left\{D_{\Psi} c\right.$ for $\left.c \in \ell^{p}\right\}$. We refer to [34] for an early example and the terminology of coorbit spaces.

\section{Localization of frames}

In this section we introduce the concept of localized frames and define the corresponding family of coorbit spaces. In Subsection 3.1 we treat the maximal space $\mathscr{H}_{w}^{\infty}$ in detail. In Subsection 3.2 we show the duality relations of these spaces . In Subsection 3.3 we study the frame-related operators.

We call a Banach $*$-algebra $\mathscr{A}$ of infinite matrices (over the index set $K$ ) a solid spectral matrix algebra, if

(i) $\mathscr{A} \subseteq \mathfrak{B}\left(\ell^{2}\right)$.

(ii) $\mathscr{A}$ is inverse-closed in $\mathfrak{B}\left(\ell^{2}\right)$, i.e., $A \in \mathscr{A}$ and $A$ is invertible on $\ell^{2}$, then $A^{-1} \in \mathscr{A}$.

(iii) $\mathscr{A}$ is solid, i.e., $A \in \mathscr{A}$ and $\left|b_{k, l}\right| \leq\left|a_{k, l}\right|$, then $B=\left(b_{k, l}\right) \in \mathscr{A}$ and $\|B\|_{\mathscr{A}} \leq\|A\|_{\mathscr{A}}$.

Several examples, e.g., the Jaffard class or a Schur-type class, can be found in [38]. In these examples localization is defined by some off-diagonal decay of the Gram matrix. For the systematic construction of spectral matrix algebras we refer to 55 , [57, 54, 76], a survey on spectral invariance is contained in [53].

Definition 1. Let $\mathscr{A}$ be solid spectral matrix algebra. Two frames $\Psi$ and $\Phi$ are called $\mathscr{A}$-localized with respect to each other, if their cross-Gram matrix $G_{\Psi, \Phi}$ belongs to $\mathscr{A}$. If $G_{\Psi, \Phi} \in \mathscr{A}$, we write $\Psi \sim_{\mathscr{A}} \Phi$. 
A single frame $\Psi=\left(\psi_{k}\right)$ is called (intrinsically) $\mathscr{A}$-localized, if $\Psi \sim \mathscr{A} \Psi$.

Alternative definitions of localized frames can be found in [39] (continuous frames), [48, 50] (localization with respect to a Riesz basis), [3] ( $\ell^{p}$-self-localization) or [44, 71, 33] (localization in terms of the intrinsic metric on a manifold). Although all these concepts have their merits, we will focus on the intrinsic localization of Definition 1 .

The following connection holds for any chosen dual frame $\Phi^{d}[38$ :

$$
\Psi \sim_{\mathscr{A}} \Phi, \Phi^{d} \sim_{\mathscr{A}} \Xi \Longrightarrow \Psi \sim_{\mathscr{A}} \Xi
$$

A weight $w$ is called $\mathscr{A}$-admissible, if every $A \in \mathscr{A}$ can be extended to a bounded operator on $\ell_{w}^{p}$ for all $1 \leq p \leq \infty$ i.e, $\mathscr{A} \subseteq \bigcap_{1 \leq p \leq \infty} \mathfrak{B}\left(\ell_{w}^{p}\right)$.

In the following, $\mathscr{A}$ is always a solid spectral Banach algebra of matrices on $K$. Since $\mathscr{A}$ is a Banach $*$-algebra, if $w$ is $\mathscr{A}$-admissible, then $1 / w$ is admissible, too. This is because for $A: \ell_{w}^{p} \rightarrow \ell_{w}^{p}$, we have $A^{*}: \ell_{1 / w}^{q} \rightarrow \ell_{1 / w}^{q}$ for $q>1$. For $q=1$, this argument is valid using the pre-dual.

Definition 2. Let $\mathscr{H}^{00}=\left\{f=\sum_{k} c_{k} \psi_{k}: c \in \ell^{00}\right\}$ be the subspace of all finite linear combinations over $\Psi$.

For $1 \leq p<\infty$ define $\mathscr{H}_{w}^{p}(\Psi, \tilde{\Psi})$ as the completion of $\mathscr{H}^{00}$ with respect to the norm

$$
\|f\|_{\mathscr{H}_{w}^{p}}=\left\|C_{\widetilde{\psi}}(f)\right\|_{\ell_{w}^{p}} .
$$

Let $\mathscr{H}_{w}^{0}$ be the completion of $\mathscr{H}^{00}$ with respect to the norm

$$
\|f\|_{\mathscr{H}_{w}^{0}}=\|f\|_{\mathscr{H}_{w}^{\infty}}=\left\|C_{\tilde{\psi}}(f)\right\|_{\ell_{w}^{\infty}} .
$$

In Section 3.1 we will define the space $\mathscr{H}_{w}^{\infty}$ as a weak ${ }^{*}$ completion with respect to the metric $\left\|C_{\tilde{\psi}}(f)\right\|_{\ell_{w}^{\infty}}$. Alternatively, we may define it as the bidual $\mathscr{H}_{w}^{\infty}=\left(\mathscr{H}_{w}^{0}\right)^{* *}$.

We note right away that $\mathscr{H}_{w}^{p} \subseteq \mathscr{H}_{w}^{q} \subseteq \mathscr{H}_{w}^{0}$ for $1 \leq p \leq q$.

As a consequence of this definition the analysis operator can be extended to a bounded operator from $\mathscr{H}_{w}^{p}$ into $\ell_{w}^{p}$.

The main results in [38] are summarized below. The first one describes the independence of $\mathscr{H}_{w}^{p}(\Psi, \tilde{\Psi})$ of the defining frame $\Psi$.

Proposition 1 ([38]). Let $\Phi$ and $\Psi$ be frames for $\mathscr{H}$ and $\Phi^{d}$ and $\Psi^{d}$ dual frames. If $\Psi^{d} \sim_{\mathscr{A}} \Psi, \Phi^{d} \sim_{\mathscr{A}} \Psi$ and $\Psi^{d} \sim_{\mathscr{A}} \Phi$, then $\mathscr{H}_{w}^{p}\left(\Psi, \Psi^{d}\right)=\mathscr{H}_{w}^{p}\left(\Phi, \Phi^{d}\right)$ with equivalent norms for all $1 \leq p \leq \infty$.

The proof of this result relies on the algebra properties of $\mathscr{A}$ and identities for Gram matrices. By this result we may therefore write unambiguously $\mathscr{H}_{w}^{p}:=$ $\mathscr{H}_{w}^{p}(\Psi, \tilde{\Psi})=\mathscr{H}_{w}^{p}(\tilde{\Psi}, \Psi)$. 
In particular, let $\Phi=\Psi$. For a frame $\Psi$ and its dual $\Psi^{d}$, which are $\mathscr{A}$-localized with respect to each other, it can be shown that they are automatically Banach frames for all involved associated Banach spaces:

Theorem 1 ([38]). Assume that $\Psi \sim \mathscr{A} \Psi^{d}$. Then $\Psi$ is a Banach frame for $\mathscr{H}_{w}^{p}\left(\Psi, \Psi^{d}\right)$ for $1 \leq p<\infty$ or $p=0$. The reconstructions $f=\sum_{n \in N}\left\langle f, \psi_{n}\right\rangle \psi_{n}^{d}$ and $f=\sum_{n \in N}\left\langle f, \psi_{n}^{d}\right\rangle \psi_{n}$ converge unconditionally for $1 \leq p<\infty$.

The assumptions of Proposition 1 can be weakened for the canonical dual frame, because it can be shown that an intrinsically localized frame is automatically localized with respect to its canonical dual. As a consequence an intrinsically localized frame is automatically a Banach frame for all associated Banach spaces. This is the main theorem about localized frames:

Theorem 2 ([38]). Let $\Psi$ be an intrinsically $\mathscr{A}$-localized frame, $\Psi \sim_{\mathscr{A}} \Psi$. Then

$$
\tilde{\Psi} \sim_{\mathscr{A}} \tilde{\Psi} \text { and } \Psi \sim_{\mathscr{A}} \tilde{\Psi} .
$$

As a consequence, $\mathscr{H}_{w}^{p}(\Psi, \tilde{\Psi})=\mathscr{H}_{w}^{p}(\tilde{\Psi}, \Psi)$ and $\Psi$ is a $p$-Banach frame for $\mathscr{H}_{w}^{p}(\Psi, \tilde{\Psi})$ for $1 \leq p<\infty$ or $p=0$. The reconstructions

$$
f=\sum_{n \in N}\left\langle f, \psi_{n}\right\rangle \tilde{\psi}_{n} \text { and } f=\sum_{n \in N}\left\langle f, \tilde{\psi}_{n}\right\rangle \psi_{n}
$$

converge unconditionally in $\mathscr{H}_{w}^{p}$ for $1 \leq p<\infty$.

Therefore the norms $\left\|C_{\Psi} f\right\|_{\ell_{w}^{p}}$ and $\left\|C_{\widetilde{\Psi}} f\right\|_{\ell_{w}^{p}}$ are equivalent, and the inequalities

$$
\frac{1}{\left\|G_{\widetilde{\Psi}}\right\|_{\ell_{w}^{p} \rightarrow \ell_{w}^{p}}}\|f\|_{\mathscr{H}_{w}^{p}} \leq\left\|C_{\Psi} f\right\|_{\ell_{w}^{p}} \leq\left\|G_{\Psi}\right\|_{\ell_{w}^{p} \rightarrow \ell_{w}^{p}}\|f\|_{\mathscr{H}_{w}^{p}} .
$$

are valid for $1 \leq p<\infty$ and $p=0$

The unconditional convergence of the reconstruction formula (6) implies that both synthesis operators $D \Psi$ and $D_{\widetilde{\Psi}}$ map $\ell_{w}^{p}$ onto $\mathscr{H}_{w}^{p}$ for $1 \leq p<\infty$ and $p=0$. Consequently, an equivalent norm on $\mathscr{H}_{w}^{p}$ is given by

$$
\inf \left\{\|c\|_{\ell_{w}^{p}}: f=D_{\psi} c\right\} \quad \text { for } f \in \mathscr{H}_{w}^{p}
$$

In particular this means that the orbit and co-orbit definitions of $\mathscr{H}_{w}^{p}$ coincide.

The best studied examples of intrinsically localized frames are the following.

(i) Frames of translates [11, 21],

(ii) Frames of reproducing kernels in a shift-invariant space [50, 77]

(iii) Gabor frames [36, 50, 38],

(iv) Frames of reproducing kernels in (general) Bargmann-Fock spaces [65],

(v) Wavelet frames that are orthogonal across different scales [21, 37]. 
However, not all useful frames are localized in the sense of Definition 1 among them are general wavelet frames, frames of curvelets, shearlets, frames on manifolds, etc. Although these frames do possess some form of localization, they are not part of our theory of localized frames. While many of the constructions discussed in this chapter, such as the definition and characterization of coorbit spaces, can be carried out by hand or with different techniques, the main results for localized frames are not available for wavelets or curvelets. For instance, the decisive Theorem 2 and most of its consequences are false for wavelet frames and their many generalizations.

\section{$3.1 \mathscr{H}_{w}^{\infty}$ as a normed space}

In the following we will focus on the theory of the coorbit spaces $\mathscr{H}_{w}^{p}(\Psi, \widetilde{\Psi})$ that are associated to a localized frame. We start with the "distribution space" $\mathscr{H}_{w}^{\infty}$ and offer a thorough treatment. In [38] "the rigorous discussion was omitted to avoid tedious technicalities."

Let $w$ be an $\mathscr{A}$-admissible weight. We define $\mathscr{H}_{w}^{\infty}$ as a certain weak ${ }^{*}$ completion of $\mathscr{H}$. We say that two sequences $\left(f_{n}\right)$ and $\left(g_{n}\right)$ in $\mathscr{H}$ are equivalent, denoted by $f_{n} \sim g_{n}$, if $\left\langle f_{n}-g_{n}, \tilde{\psi}_{k}\right\rangle \rightarrow 0$ as $n \rightarrow \infty$. Alternatively, $f_{n}-g_{n} \rightarrow 0$ in the $\sigma\left(\mathscr{H}, \mathscr{H}^{00}\right)$-topology.

Definition 3. We define $\mathscr{H}_{w}^{\infty}$ as the set of equivalence classes of sequences $f=\left[f_{n}\right]$, such that

(i) $f_{n} \in \mathscr{H}$ for all $n \in \mathbb{N}$,

(ii) $\lim _{n \rightarrow \infty}\left\langle f_{n}, \tilde{\psi}_{k}\right\rangle=\alpha_{k}$ exists for all $k \in K$,

(iii) $\sup _{n}\left\|C_{\widetilde{\Psi}} f_{n}\right\|_{\ell_{w}^{\infty}}<\infty$.

In this way $\mathscr{H}_{w}^{\infty}$ is well-defined. The definition of $f$ is independent of its representative. Indeed, if $f=\left[f_{n}\right]$ and $f_{n} \sim g_{n}$, then $\alpha_{k}=\lim _{n \rightarrow \infty}\left\langle f_{n}, \tilde{\psi}_{k}\right\rangle=$ $\lim _{n \rightarrow \infty}\left\langle g_{n}, \tilde{\psi}_{k}\right\rangle$.

Furthermore, condition (iii) implies that $\left|\left\langle f_{n}, \tilde{\psi}_{k}\right\rangle\right| w_{k} \leq C$ for all $n \in \mathbf{N}$ and $k \in$ $K$, consequently, $\left|\alpha_{k}\right| w_{k}=\lim _{n \rightarrow \infty}\left|\left\langle f_{n}, \tilde{\psi}_{k}\right\rangle\right| w_{k} \leq C$ and thus $\alpha \in \ell_{w}^{\infty}$. Now, write $\left\langle f, \tilde{\psi}_{k}\right\rangle=\alpha_{k}$. and set

$$
\|f\|_{\mathscr{H}_{w}^{\infty}}=\|\alpha\|_{\ell_{w}^{\infty}} .
$$

Therefore $C_{\widetilde{\Psi}}: \mathscr{H}_{w}^{\infty} \rightarrow \ell_{w}^{\infty}$ is a bounded operator.

Clearly, (8) defines a seminorm, because limits are linear and $\|\cdot\|_{\ell_{w}^{\infty}}$ is a norm. Now assume that $\|f\|_{\mathscr{H}_{w}^{\infty}}=0$. This means that for every representative $\left[f_{n}\right]$ of $f$ we have $\lim _{n}\left\langle f_{n}, \tilde{\psi}_{k}\right\rangle=0$, or equivalently $f_{n} \sim 0$. Thus $f=0$ in $\mathscr{H}$ and $\|\cdot\|_{\mathscr{H}_{w}^{\infty}}$ is indeed a norm.

Lemma 1. (i) The map $f=\left[f_{n}\right] \mapsto \alpha=\left(\lim _{n}\left\langle f_{n}, \tilde{\psi}_{k}\right\rangle\right)_{k \in K}$ is an isometric isomorphism from $\mathscr{H}_{w}^{\infty}$ onto the subspace $V_{\Psi}=\left\{\alpha \in \ell_{w}^{\infty}: \alpha=G_{\tilde{\Psi}, \Psi)} \alpha\right\}$. 
(ii)The subspace $V_{\Psi}$ is closed in $\ell_{w}^{\infty}$ and thus $\mathscr{H}_{w}^{\infty}$ is complete.

Proof. (i) For $f \in \mathscr{H}$ we interpret the reconstruction formula $f=\sum_{l}\left\langle f, \tilde{\psi}_{l}\right\rangle \psi_{l}$ weakly as $\sum_{l}\left\langle f, \tilde{\psi}_{l}\right\rangle\left\langle\psi_{l}, \tilde{\psi}_{k}\right\rangle$, or in operator notation as

$$
C_{\tilde{\Psi}} f=G_{\tilde{\Psi}, \Psi} C_{\tilde{\Psi}} f .
$$

Now let $f=\left[f_{n}\right] \in \mathscr{H}_{w}^{\infty}$ as in Definition 3 This is a sequence of vectors $f_{n} \in \mathscr{H}$ such that $\lim _{n \rightarrow \infty}\left\langle f_{n}, \tilde{\psi}_{k}\right\rangle=\alpha_{k}$ and $\left\|C_{\tilde{\Psi}} f_{n}\right\|_{\ell_{w}^{\infty}} \leq C$ for all $n$. This means that the sequence $C_{\tilde{\Psi}} f_{n}$ converges pointwise to $\alpha$ and is dominated by the sequence $C\left(w_{l}^{-1}\right)_{l} \in \ell_{w}^{\infty}$. By dominated convergence it now follows that (again with pointwise convergence)

$$
\alpha=\lim _{n \rightarrow \infty} C_{\widetilde{\Psi}} f_{n}=\lim _{n \rightarrow \infty} G_{\widetilde{\Psi}, \Psi} C_{\tilde{\Psi}} f_{n}=G_{\widetilde{\Psi}, \Psi} \lim _{n \rightarrow \infty} C_{\widetilde{\Psi}} f_{n}=G_{\widetilde{\Psi}, \Psi} \alpha .
$$

Consequently, the limiting sequence $\alpha \in \ell_{w}^{\infty}$ satisfies $\alpha=G_{\tilde{\Psi}, \Psi} \alpha$ and $\alpha \in V_{\Psi}$.

Conversely, let $\alpha \in V_{\Psi}$. Choose a sequence $F_{n}$ of finite subsets of $K$, such that $F_{n} \subset F_{n+1}$ and $\bigcup_{n=1}^{\infty} F_{n}=K$ and define

$$
f_{n}=\sum_{l \in F_{n}} \alpha_{l} \psi_{l} \in \mathscr{H}
$$

Then clearly

$$
\lim _{n \rightarrow \infty}\left\langle f_{n}, \tilde{\psi}_{k}\right\rangle=\lim _{n \rightarrow \infty} \sum_{l \in F_{n}} \alpha_{l} \psi_{l}=G_{\tilde{\Psi}, \Psi} \alpha=\alpha,
$$

and $\sup _{k}\left|\left\langle f_{n}, \tilde{\psi}_{k}\right\rangle\right| w_{k} \leq C\|\alpha\|_{\ell_{w}^{\infty}}$. This means that $f=\left[f_{n}\right] \in \mathscr{H}_{w}^{\infty}$, and as a consequence the map $\left[f_{n}\right] \in \mathscr{H}_{w}^{\infty} \mapsto \alpha \in V_{\Psi}$ is an isometric isomorphism.

(ii) Assume that $\alpha_{n} \in V_{\Psi}$ and $\alpha \in \ell_{w}^{\infty}$ such that $\left\|\alpha_{n}-\alpha\right\|_{\ell_{w}^{\infty}} \rightarrow 0$. Since $G_{\tilde{\Psi}, \Psi}$ is bounded on $\ell_{w}^{\infty}$, we obtain that $\alpha=\lim _{n \rightarrow \infty} \alpha_{n}=\lim _{n} G_{\tilde{\Psi}, \Psi} \alpha_{n}=G_{\tilde{\Psi}, \Psi} \alpha$, whence $\alpha \in V_{\Psi}$ and $V_{\Psi}$ is a (norm)-closed subspace of $\ell_{w}^{\infty}$. By the identification proved in (i), $\mathscr{H}_{w}^{\infty}$ is therefore complete.

Switching the roles of $\Psi$ and $\widetilde{\Psi}$ in Definition 3 , we see that $C_{\Psi}$ is an isometry between $\mathscr{H}_{w}^{p}(\widetilde{\Psi}, \Psi)$ and a closed subspace of $\ell_{w}^{\infty}$. By Equation (7) the corresponding norms are equivalent and so $C_{\Psi}$ is bounded from $\mathscr{H}_{w}^{p}$ into $\ell_{w}^{\infty}$.

We next verify the unconditional weak *-convergence of the sum $D_{\Psi} c=$ $\sum_{k \in K} c_{k} \psi_{k}$ for $\left(c_{k}\right) \in \ell_{w}^{\infty}$. Let $\varepsilon>0$ and $g \in \mathscr{H}^{00}$. Choose a finite set $H_{0}$, such that $\sum_{k \notin H_{0}}\left|\left\langle g, \psi_{k}\right\rangle\right| w_{k}^{-1}<\varepsilon /\|c\|_{\ell_{w}^{\infty}}$. Now let $H, J \subseteq K$ be two finite sets such that $H \supseteq H_{0}$ and $J \supseteq H_{0}$. Then $H \backslash J \cup J \backslash H \subseteq K \backslash H_{0}$, and therefore

$$
\begin{gathered}
\left|\left\langle\sum_{k \in J} c_{k} \psi_{k}-\sum_{k \in H} c_{k} \psi_{k}, g\right\rangle\right|=\left|\left\langle\sum_{k \in H \backslash J \cup J \backslash H} c_{k} \psi_{k}, g\right\rangle\right| \\
\leq\|c\|_{\ell_{w}^{\infty}} \sum_{k \notin H_{0}}\left|\left\langle\psi_{k}, g\right\rangle\right| w_{k}^{-1}<\varepsilon .
\end{gathered}
$$

Thus the series for $D_{\Psi} c$ converges weak-* unconditionally. Furthermore, since $G_{\tilde{\Psi}, \Psi}$ is bounded on $\ell_{w}^{\infty}$ by the basic assumption on $\Psi$ and Theorem 2 , we also deduce the 
boundedness of $D_{\Psi}$ as follows:

$$
\|D \Psi c\|_{\mathscr{H}_{w}^{\infty}}=\left\|C_{\tilde{\Psi}} D \Psi c\right\|_{\ell_{w}^{\infty}}=\left\|G_{\tilde{\Psi}, \Psi} c\right\|_{\ell_{w}^{\infty}} \leq\left\|G_{\tilde{\Psi}, \Psi}\right\|_{\ell_{w}^{\infty} \rightarrow \ell_{w}^{\infty}}\|c\|_{\ell_{w}^{\infty}}<\infty .
$$

The following lemma summarizes the properties of $\mathscr{H}_{w}^{\infty}$.

Lemma 2. Let $\Psi$ be an $\mathscr{A}$-localized frame and $w$ an $\mathscr{A}$-admissible weight. Then $\left(\mathscr{H}_{w}^{\infty},\|\cdot\|_{\mathscr{H}_{w}^{\infty}}\right)$ is a Banach space, and

(i) $C_{\Psi}:\left(\mathscr{H}_{w}^{\infty},\|\cdot\|_{\mathscr{H}_{w}^{\infty}}\right) \rightarrow \ell_{w}^{\infty}$ is continuous.

(ii) $D \Psi: \ell_{w}^{\infty} \rightarrow\left(\mathscr{H}_{w}^{\infty},\|\cdot\|_{\mathscr{H}_{w}^{\infty}}\right)$ is continuous with $\|D \Psi\|_{\ell_{w}^{\infty} \rightarrow \mathscr{H}_{w}^{\infty}} \leq\left\|G_{\tilde{\Psi}, \Psi}\right\|_{\ell_{w}^{\infty} \rightarrow \ell_{w}^{\infty}}$.

The series $D c=\sum_{k \in K} c_{k} \psi_{k}$ is weak-* unconditionally convergent.

\subsection{Duality}

The associated Banach spaces $\mathscr{H}_{w}^{p}$ are a generalization of the coorbit spaces in [34] and the modulation spaces [32]. We first formulate their duality.

Proposition 2. Let $\Psi$ be a $\mathscr{A}$-localized frame and $w$ an admissible weight. Let $1 \leq$ $p<\infty$ and $q$ such that $\frac{1}{p}+\frac{1}{q}=1$ or $(p, q)=(0,1)$. Then

$$
\left(\mathscr{H}_{w}^{p}\right)^{\prime} \cong \mathscr{H}_{1 / w}^{q}
$$

where the duality for $f \in \mathscr{H}_{w}^{p}$ and $h \in \mathscr{H}_{1 / w}^{q}$ is given by

$$
\langle f, h\rangle_{\mathscr{H}_{w}^{p}, \mathscr{H}_{1 / w}^{q}}:=\left\langle C_{\widetilde{\Psi}} f, C_{\Psi} h\right\rangle_{\ell_{w}^{p}, \ell_{1 / w}^{q}} .
$$

Proof. Fix $h \in \mathscr{H}_{1 / w}^{q}$. Then, using the duality of $\ell_{w}^{p}$ and $\ell_{1 / w}^{q}$, we define a sesquilinear form by

$$
\langle f, h\rangle_{\mathscr{H}_{w}^{p}, \mathscr{H}_{1 / w}^{q}}=\left\langle C_{\widetilde{\Psi}} f, C_{\Psi} h\right\rangle_{\ell_{w}^{p}, \ell_{1 / w}^{q}},
$$

for $f \in \mathscr{H}_{w}^{p}$.

Now set $\mathscr{W}(h)(f)=\langle f, h\rangle_{\mathscr{H}_{w}^{p}, \mathscr{H}_{1 / w}^{q}}$. Then $|\mathscr{W}(h)(f)| \leq\left\|C_{\widetilde{\Psi}} f\right\|_{\ell_{w}^{p}}\left\|C_{\Psi} h\right\|_{\ell_{1 / w}^{q}}=$ $\left\|C_{\Psi} h\right\|_{\ell_{1 / w}^{q}} \cdot\|f\|_{\mathscr{H}_{w}^{p}}$, since $C_{\Psi} h \in \ell_{1 / w}^{q}$ by the norm equivalence (7). If $p=0$, we use the estimate $|\mathscr{W}(h)(f)| \leq\left\|C_{\widetilde{\Psi}} f\right\|_{\ell_{w}^{\infty}}\left\|C_{\Psi} h\right\|_{\ell_{1 / w}^{1}}=\left\|C_{\Psi} h\right\|_{\ell_{1 / w}^{1}} \cdot\|f\|_{\mathscr{H}_{w}^{0}}$, Therefore $\mathscr{W}(h) \in\left(\mathscr{H}_{w}^{p}\right)^{\prime}$. As a consequence $\mathscr{W}: \mathscr{H}_{1 / w}^{q} \rightarrow\left(\mathscr{H}_{w}^{p}\right)^{\prime}$ is bounded, with $\|\mathscr{W}(h)\|_{\left(\mathscr{H}_{w}^{p}\right)^{\prime}} \leq\left\|C_{\Psi} h\right\|_{\ell_{1 / w}^{q}}$.

Conversely, let $H \in\left(\mathscr{H}_{w}^{p}\right)^{\prime}$ and $c \in \ell_{w}^{p}$ be arbitrary with $1 \leq p \leq \infty$ or $p=0$. Then $D_{\Psi c} c$ is in $\mathscr{H}_{w}^{p}$ and so $H\left(D_{\Psi c}\right)=\sum_{k} c_{k} H\left(\psi_{k}\right)$ with unconditional conver- 
gence. Therefore the sequence $\left(H\left(\psi_{k}\right)\right)_{k}$ is in $\ell_{1 / w}^{q}$ [18]. Now define the operator $\mathscr{V}:\left(\mathscr{H}_{w}^{p}\right)^{\prime} \rightarrow \mathscr{H}_{1 / w}^{q}$ by $\mathscr{V}(H)=\sum_{k} \overline{H\left(\psi_{k}\right)} \tilde{\psi}_{k}$.

For $f \in \mathscr{H}_{w}^{p}$ we have

$$
\begin{gathered}
\mathscr{W}(\mathscr{V}(H))(f)=\left\langle C_{\widetilde{\Psi}} f, C_{\Psi} \mathscr{V}(H)\right\rangle_{\ell_{w}^{p}, \ell_{1 / w}^{q}}=\sum_{l}\left\langle f, \tilde{\psi}_{l}\right\rangle \overline{\left\langle\sum_{k} \overline{H\left(\psi_{k}\right)} \tilde{\psi}_{k}, \psi_{l}\right\rangle}= \\
=\sum_{k} H\left(\psi_{k}\right)\left\langle\sum_{l}\left\langle f, \tilde{\psi}_{l}\right\rangle \psi_{l}, \tilde{\psi}_{k}\right\rangle=\sum_{k} H\left(\psi_{k}\right)\left\langle f, \tilde{\psi}_{k}\right\rangle=H\left(\sum_{k}\left\langle f, \tilde{\psi}_{k}\right\rangle \psi_{k}\right)=H(f) .
\end{gathered}
$$

The formal manipulations are justified by the unconditional convergence of the series involved, by the continuity of $H$, and by density arguments. Thus $\mathscr{W}: \mathscr{H}_{1 / w}^{q} \rightarrow$ $\left(\mathscr{H}_{w}^{p}\right)^{\prime}$ is onto.

On the other hand

$$
\begin{gathered}
\mathscr{V}(\mathscr{W}(h))=\sum_{l} \overline{\mathscr{W}(h)\left(\psi_{l}\right)} \tilde{\psi}_{l}=\sum_{l} \overline{\sum_{k}\left\langle\psi_{l}, \tilde{\psi}_{k}\right\rangle \overline{\left\langle h, \psi_{k}\right\rangle}} \tilde{\psi}_{l}= \\
=\sum_{k}\left\langle h, \psi_{k}\right\rangle\left(\sum_{l}\left\langle\tilde{\psi}_{k}, \psi_{l}\right\rangle \tilde{\psi}_{l}\right)=\sum_{k}\left\langle h, \psi_{k}\right\rangle \tilde{\psi}_{k}=h .
\end{gathered}
$$

Therefore $\mathscr{W}$ is invertible.

Similar results appeared in [34] and [27].

Remark 1 . Note that the duality is consistent with the inner product $\langle.,$.$\rangle on \mathscr{H}$, see Lemma4

Also, note that the isomorphism $\left(\mathscr{H}_{w}^{p}\right)^{\prime} \cong \mathscr{H}_{1 / w}^{q}$ is not an isometric isomorphism.

By the above result we now have $\left(\mathscr{H}_{1 / w}^{1}\right)^{\prime} \cong\left(\mathscr{H}_{w}^{\infty},\|\cdot\|_{\mathscr{H}_{w}^{\infty}}\right)$. This yields another proof for the completeness of $\left(\mathscr{H}_{w}^{\infty},\|\cdot\|_{\mathscr{H}_{w}^{\infty}}\right)$.

\subsubsection{Duality for $\mathscr{H}_{w}^{\infty}$}

For $p=\infty$ we can now prove a reconstruction result, as an extension to Theorem 1

Lemma 3. Let $\Psi$ be a $\mathscr{A}$-localized frame and $w$ an $\mathscr{A}$-admissible weight. If $f \in \mathscr{H}_{w}^{\infty}$, then $f=\sum\left\langle f, \psi_{k}\right\rangle \tilde{\psi}_{k}$ and $f=\sum\left\langle f, \tilde{\psi}_{k}\right\rangle \psi_{k}$ with weak-* unconditional convergence in $\sigma\left(\mathscr{H}_{w}^{\infty}, \mathscr{H}_{1 / w}^{1}\right)$.

Therefore $f=D_{\widetilde{\Psi}} C_{\Psi} f=D_{\Psi} C_{\tilde{\Psi}} f$, and, in particular $D_{\widetilde{\Psi}}$ and $D \Psi$ are onto $\mathscr{H}_{w}^{\infty}$.

The norm equivalence (7) is valid for all $1 \leq p \leq \infty$ and $p=0$ :

$$
\frac{1}{\left\|G_{\widetilde{\Psi}}\right\|_{\ell_{w}^{p} \rightarrow \ell_{w}^{p}}}\|f\|_{\mathscr{H}_{w}^{p}} \leq\left\|C_{\Psi} f\right\|_{\ell_{w}^{p}} \leq\left\|G_{\Psi}\right\|_{\ell_{w}^{p} \rightarrow \ell_{w}^{p}}\|f\|_{\mathscr{H}_{w}^{p}} .
$$


Proof. By above, $\mathscr{H}_{1 / w}^{1}$ is the predual of $\mathscr{H}_{w}^{\infty}$. Now, let $f \in \mathscr{H}_{w}^{\infty}$ and $g \in \mathscr{H}_{1 / w}^{1}$, then we have

$$
\left|\sum_{k \in K}\left\langle f, \psi_{k}\right\rangle\left\langle\tilde{\psi}_{k}, g\right\rangle\right| \leq \sum_{k \in K}\left|\left\langle f, \psi_{k}\right\rangle\right|\left|\left\langle\tilde{\psi}_{k}, g\right\rangle\right| \leq\left\|C_{\Psi} f\right\|_{\ell_{w}^{\infty}}\left\|C_{\widetilde{\Psi}} g\right\|_{\ell_{1 / w}^{1}},
$$

and the sum of the left-hand side converges absolutely.

By Lemma $22 D_{\tilde{\Psi}} C_{\Psi}$ is well-defined on all of $\mathscr{H}_{w}^{\infty}$. Let $g \in \mathscr{H}^{00}$, then

$$
\left\langle D_{\tilde{\Psi}} C \Psi f, g\right\rangle=\lim _{\substack{H \rightarrow K \\ H \text { finite }}}\left\langle\sum_{k \in H}\left\langle f, \psi_{k}\right\rangle \cdot \tilde{\psi}_{k}, g\right\rangle=\lim _{\substack{H \rightarrow K \\ H \text { finite }}} \sum_{k \in H}\left\langle f, \psi_{k}\right\rangle \cdot\left\langle\tilde{\psi}_{k}, g\right\rangle=\langle f, g\rangle .
$$

And so $f=D_{\tilde{\Psi}} C_{\Psi} f$.

The second reconstruction formula follows by an analogous argument. The norm equivalence 170 follows immediately from the reconstruction formula.

We can formulate the compatibility of the duality relations in the following way.

Lemma 4. Let $\Psi$ be a $\mathscr{A}$-localized frame and $w$ an admissible weight. For $f \in \mathscr{H}_{w}^{p}$ and $h \in \mathscr{H}_{1 / w}^{1}$ we have

$$
\langle f, h\rangle_{\mathscr{H}_{w}^{p}, \mathscr{H}_{1 / w}^{q}}=\langle f, h\rangle_{\mathscr{H}_{w}^{\infty}, \mathscr{H}_{1 / w}^{1}} \cdot
$$

Proof. The identity follows from the definition of the duality in Proposition 2 because

$$
\langle f, h\rangle_{\mathscr{H}_{w}^{p}, \mathscr{H}_{1 / w}^{q}}=\left\langle C_{\widetilde{\Psi}} f, C_{\Psi} h\right\rangle_{\ell_{w}^{p}, \ell_{1 / w}^{q}}=\left\langle C_{\widetilde{\Psi}} f, C_{\Psi} h\right\rangle_{\ell_{w}^{\infty}, \ell_{1 / w}^{1}}=\langle f, h\rangle_{\mathscr{H}_{w}^{\infty}, \mathscr{H}_{1 / w}^{1}} .
$$

After clarifying the meaning of the duality brackets, we can now give the traditional definition of the coorbit space $\mathscr{H}_{w}^{p}$ as a subspace of "distributions".

Proposition 3. Let $\Psi$ be an $\mathscr{A}$-localized frame and $w$ an admissible weight. For $1 \leq p<\infty$ we have

$$
\mathscr{H}_{w}^{p}=\left\{f \in \mathscr{H}_{w}^{\infty}: f=\sum_{k \in K}\left\langle f, \tilde{\psi}_{k}\right\rangle_{\mathscr{H}_{w}^{\infty}, \mathscr{H}_{1 / w}^{1}} \psi_{k} \text { with }\left\langle f, \tilde{\psi}_{k}\right\rangle_{\mathscr{H}_{w}^{\infty}, \mathscr{H}_{1 / w}^{1}} \in \ell_{w}^{p}\right\},
$$

with unconditional convergence.

Proof. We combine the reconstruction formula in Theorem 2 with the identities $\left(\left\langle f, \psi_{k}\right\rangle_{\mathscr{H}_{w}^{\infty}, \mathscr{H}_{1 / w}^{1}}\right)_{k \in K}=\left(\left\langle f, \psi_{k}\right\rangle_{\mathscr{H}_{w}^{p}, \mathscr{H}_{1 / w}^{q}}\right)_{k \in K} \in \ell_{w}^{p}$ from Lemma 4 and use the unconditional convergence in $\mathscr{H}_{w}^{p}$. We obtain

$$
f=\sum_{k \in K}\left\langle f, \psi_{k}\right\rangle_{\mathscr{H}_{w}^{p}, \mathscr{H}_{1 / w}^{q}} \tilde{\psi}_{k}=\sum_{k \in K}\left\langle f, \psi_{k}\right\rangle_{\mathscr{H}_{w}^{\infty}, \mathscr{H}_{1 / w}^{1}} \tilde{\psi}_{k} .
$$


For $p=\infty$ we can state the following characterization (using Proposition 2 and Lemma3):

Corollary 1. Let $\Psi$ be a $\mathscr{A}$-localized frame. Let $W$ and $w$ admissible weights satisfying $W \leq w$ so that $\mathscr{H}_{w}^{\infty} \subseteq \mathscr{H}_{W}^{\infty}$. Then for $f \in \mathscr{H}_{W}^{\infty}$ the following properties are equivalent:

(i) $f \in \mathscr{H}_{w}^{\infty}$.

(ii) $\left\|C_{\widetilde{\Psi}} f\right\|_{\ell_{w}^{\infty}}<\infty$.

(iii) There is a $c \in \ell_{w}^{\infty}$, such that $f=\sum_{k \in K} c_{k} \psi_{k}$ with $C_{\widetilde{\Psi}} f=G_{\Psi, \widetilde{\Psi}} c$ and

$$
\left\|C_{\widetilde{\Psi}} f\right\|_{\ell_{w}^{\infty}}=\left\|G_{\Psi, \widetilde{\Psi}} c\right\|_{\ell_{w}^{\infty}} .
$$

(iv) $f \in\left(\mathscr{H}_{w}^{1}\right)^{\prime}$.

\subsubsection{The chain of Banach spaces $\mathscr{H}_{w}^{p}$}

Formulated for Gelfand triples we obtain the following consequence.

Corollary 2. Let $\Psi$ be a $\mathscr{A}$-localized frame and $w$ an admissible weight with $\inf _{k \in K} w_{k}>0$. Let $1 \leq p<2$ and $1 / p+1 / q=1$, or $(p, q)=(0,1)$. Then $\Psi$ is a Gelfand frame for the Gelfand triples

$$
\mathscr{H}_{w}^{p} \subseteq \mathscr{H} \subseteq \mathscr{H}_{1 / w}^{q}
$$

with respect to the duality $\left\langle C_{\widetilde{\Psi}} f, C_{\Psi} h\right\rangle_{\ell_{w}^{p}, \ell_{1 / w}^{q}}$ and the sequence spaces $\ell_{w}^{p} \subseteq \ell^{2} \subseteq$ $\ell_{1 / w^{*}}^{q}$

Proof. By Proposition $2\left(\mathscr{H}_{w}^{p}\right)^{\prime} \cong \mathscr{H}_{1 / w}^{q}$. Since $w_{k} \geq C$ we have, for $1 \leq p \leq 2$ and $2 \leq q \leq \infty$, the following inclusions

$$
\mathscr{H}_{w}^{1} \subseteq \mathscr{H}_{w}^{p} \subseteq \mathscr{H}_{w}^{2} \subseteq \mathscr{H} \subseteq \mathscr{H}_{1 / w}^{2} \subseteq \mathscr{H}_{1 / w}^{q} \subseteq \mathscr{H}_{1 / w}^{\infty},
$$

For $q<\infty$, these inclusions are norm-dense, continuous embeddings (by the corresponding inclusions (11) for sequence spaces), for $q=\infty, \mathscr{H}$ is w*-dense in $\mathscr{H}_{1 / w}^{\infty}$. Theorem 1 asserts that $\Psi$ is a Banach frame for $\mathscr{H}_{w}^{p}$ and $\mathscr{H}_{1 / w}^{q}$.

To summarize the "size" of the coorbit spaces for $1 \leq p \leq 2$ and $2 \leq q \leq \infty$ by Equation (1) we obtain the following inclusions: 


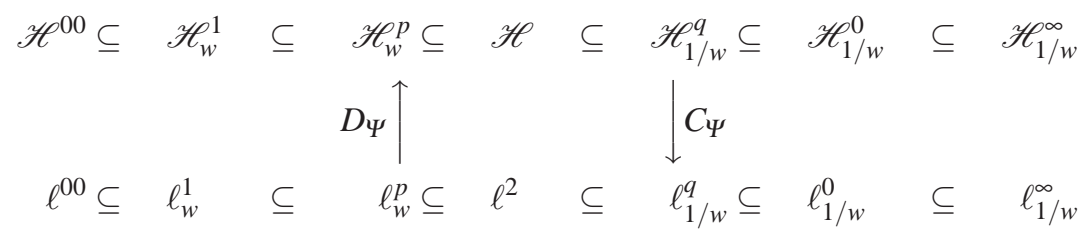

All the inclusions but the last one are in fact norm-dense embeddings; $\mathscr{H}^{00}$ is norm dense in $\mathscr{H}_{w}^{p}$ for $1 \leq p<\infty$ and weak*-dense in $\mathscr{H}_{w}^{\infty}$.

Finally we mention that the assumption on the weight serves only to obtain a "small space" with $p=1$ on the left side of the diagram. By contrast, if $1 / w \subseteq \ell^{2}$, then $\ell_{w}^{\infty} \subset \ell^{2}$, and one obtains the Gelfand pair $\mathscr{H}_{w}^{0} \subseteq \mathscr{H} \subseteq \mathscr{H}_{1 / w}^{1}$, which looks a bit unusual.

\subsubsection{Equivalence result on inclusion of sequence spaces and associated Banach spaces}

Whereas the inclusions of the coorbit spaces $\mathscr{H}_{w}^{p}$ follow from the inclusions of the weighted $\ell^{p}$-spaces, the converse is less obvious and requires more tools.

Theorem 3. Let $\Psi$ be an $\mathscr{A}$-localized norm-bounded frame, i.e., $\inf _{k}\left\|\psi_{k}\right\|_{\mathscr{H}}>0$. Let $1 \leq p_{1}, p_{2} \leq \infty$ and let $w_{1}, w_{2}$ be admissible weights. Then

$$
\mathscr{H}_{w_{1}}^{p_{1}} \subseteq \mathscr{H}_{w_{2}}^{p_{2}} \Longleftrightarrow \ell_{w_{1}}^{p_{1}} \subseteq \ell_{w_{2}}^{p_{2}}
$$

Proof. The implication $\Longleftarrow$ is clear.

Conversely, assume that $\mathscr{H}_{w_{1}}^{p_{1}} \subseteq \mathscr{H}_{w_{2}}^{p_{2}}$ and that $\ell_{w_{1}}^{p_{1}} \not \subset \ell_{w_{2}}^{p_{2}}$.

Since $\Psi$ is a frame, by the Feichtinger conjecture [13] proved in [68], $\Psi$ is a finite union of Riesz sequences. In particular, $\mathscr{H}$ contains an infinite-dimensional subspace with a Riesz basis $\Psi_{0}=\left\{\psi_{k} \mid k \in K_{0}\right\} \subseteq \Psi$.

The Gram matrix $G_{\Psi_{0}}$ is invertible on $\ell^{2}\left(K_{0}\right)$ [16]. We extend $G_{\Psi_{0}}$ to a matrix on $\ell^{2}(K)$ by defining $G c=0$ for $c \in \ell^{2}\left(K \backslash K_{0}\right)=\ell^{2}\left(K_{0}\right)^{\perp}$. Note that $G$ is obtained from the Gram matrix $G_{\Psi}$ by setting $\left(G_{\Psi}\right)_{j, k}=0$ for $j, k \notin K_{0}$. Since $\mathscr{A}$ is solid, we conclude that $G$ is in $\mathscr{A}$, and since $\mathscr{A}$ is closed with respect to the pseudo-inversion [38], we also find that $G^{\dagger} \in \mathscr{A}$. The matrix $G$ possesses the pseudo-inverse $G^{\dagger}$ with $G^{\dagger}=G_{\Psi_{0}}^{-1} c$ for $c \in \ell^{2}\left(K_{0}\right)$ and $G^{\dagger} c=0$ for $c \in \ell^{2}\left(K_{0}\right)^{\perp}$.

Extend $c \in \ell_{w_{1}}^{p_{1}}\left(K_{0}\right) \backslash \ell_{w_{2}}^{p_{2}}\left(K_{0}\right)$ to a sequence $\tilde{c} \in \ell_{w_{1}}^{p_{1}}(K) \backslash \ell_{w_{2}}^{p_{2}}(K)$ (by setting $\tilde{c}_{k}=$ 0 for $k \in K \backslash K_{0}$ ) and set $d=G^{\dagger} \tilde{c}$. In particular, $d_{k}=0$ for $k \notin K_{0}$. Since $G^{\dagger} \in \mathscr{A}$ is bounded on $\ell_{w_{1}}^{p_{1}}$, it follows that $d \in \ell_{w_{1}}^{p_{1}}(K) \backslash \ell_{w_{2}}^{p_{2}}(K)$. Therefore $f=\sum_{k \in K} d_{k} \psi_{k} \in \mathscr{H}_{w_{1}}^{p_{1}}$.

Furthermore

$$
\left(C_{\Psi_{0}} f\right)_{k}=\left\langle f, \psi_{k}\right\rangle=\sum_{l \in K_{0}} d_{l}\left\langle\psi_{l}, \psi_{k}\right\rangle=(G d)_{k}=c_{k} \text { for } k \in K_{0} .
$$


If $f \in \mathscr{H}_{w_{1}}^{p_{1}}$, then $C_{\Psi} f \in \ell_{w_{1}}^{p_{1}}$. But $\left.C_{\Psi} f\right|_{K_{0}}=c \notin \ell_{w_{2}}^{p_{2}}\left(K_{0}\right)$, and so $C_{\Psi} f \notin \ell_{w_{2}}^{p_{2}}$. Therefore $f \notin \mathscr{H}_{w_{2}}^{p_{2}}$, which is a contradiction.

Most likely, the statement could be proved without the full strength of the theorem of Marcus, Spielman, and Srivastava [68].

\subsection{Properties of the frame-related operators}

We next summarize the properties of the canonical operators associated to every frame. We include the statements for $p=\infty$ and discuss the convergence of series expansions. As a novelty, we discuss all operators with respect to a frame $\Phi$ in the same localization class, i.e. $\Phi \sim_{\mathscr{A}} \Psi$. Being pedantic, we always consider the operator as a mapping with a domain. For instance, whereas the synthesis operator is the formula $D \Psi c=\sum_{k \in K} c_{k} \psi_{k}$ we will use the notation $D_{\Psi}^{p, w}$ to denote the synthesis operator on $\mathscr{H}_{w}^{p}$.

Theorem 4. Let $\Psi$ be an $\mathscr{A}$-localized frame and let $w$ be an $\mathscr{A}$-admissible weight. Let $1 \leq p \leq \infty$ and let $1 / p+1 / q=1$ or $(p, q)=(0,1)$.

(i) The analysis operator $C_{\Psi}^{p, w}: \mathscr{H}_{w}^{p} \rightarrow \ell_{w}^{p}$ is given by

$$
C_{\Psi}^{p, w} f=\left(\left\langle f, \psi_{k}\right\rangle_{\mathscr{H}_{w}^{p}, \mathscr{H}_{1 / w}^{q}}, k \in K\right)
$$

Then $C_{\Psi}^{p, w}$ is bounded, one-to-one and has closed range in $\ell_{w}^{p}$. $C_{\Psi}^{p, w}$ is the restric-

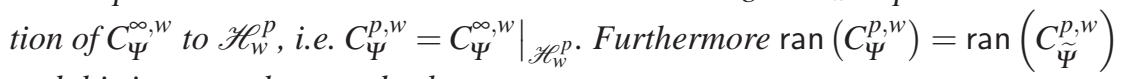
and this is a complemented subspace:

$$
\ell_{w}^{p}=\operatorname{ran}\left(C_{\Psi}^{p, w}\right) \oplus \operatorname{ker}\left(D_{\Psi}^{p, w}\right),
$$

$\Psi$ is an $\ell_{w}^{p}$-Banach frame for all $\mathscr{H}_{w}^{p}$ with bounds

$$
A=\left\|G_{\widetilde{\Psi}}^{p, w}\right\|_{\ell_{w}^{p} \rightarrow \ell_{w}^{p}}^{-1} \text { and } B=\left\|G_{\Psi}^{p, w}\right\|_{\ell_{w}^{p} \rightarrow \ell_{w}^{p}} .
$$

(ii) The synthesis (or reconstruction) operator $D_{\Psi}^{p, w}: \ell_{w}^{p} \rightarrow \mathscr{H}_{w}^{p}$ is given by

$$
D_{\Psi}^{p, w} c=\sum_{k} c_{k} \psi_{k}
$$

with unconditional convergence in $\mathscr{H}_{w}^{p}$ for $1 \leq p<\infty$ and $p=0$, and weak $*_{-}$ convergence for $p=\infty$. Then $D_{\psi}^{p, w}$ is bounded with operator norm 1 and it maps onto $\mathscr{H}_{w}^{p}$. Furthermore $D_{\Psi}^{p, w}$ is the restriction of $D_{\Psi}^{\infty, w}$ to $\mathscr{H}_{w}^{p}$, i.e. $D_{\Psi}^{p, w}=$ $\left.D_{\Psi}^{\infty, w}\right|_{\mathscr{H}_{w}^{p}}$. For $p<\infty$ 
Localized Frames and Galerkin

$$
\begin{aligned}
& \left(D_{\Psi}^{p, w}\right)^{*}=C_{\Psi}^{q, 1 / w}, \text { and } \\
& \left(C_{\Psi}^{p, w}\right)^{*}=D_{\Psi}^{q, 1 / w} .
\end{aligned}
$$

(iii) The frame operator $S_{\Psi}^{p, w}: \mathscr{H}_{w}^{p} \rightarrow \mathscr{H}_{w}^{p}$ is defined by

$$
S_{\Psi}^{p, w} f=\sum_{k}\left\langle f, \psi_{k}\right\rangle_{\mathscr{H}_{w}^{p}, \mathscr{H}_{1 / w}^{q}} \cdot \psi_{k}=\sum_{k}\left\langle f, \psi_{k}\right\rangle_{\mathscr{H}_{w}^{\infty}, \mathscr{H}_{1 / w}^{1}} \cdot \psi_{k}
$$

with unconditional convergence in $\mathscr{H}_{w}^{p}$ for $1 \leq p<\infty$ and $p=0$. It is unconditionally weak ${ }^{*}$-convergent for $p=\infty$. Furthermore $S_{\Psi}^{p, w}=D_{\Psi}^{p, w} C_{\Psi}^{p, w}$ and $\left(S_{\Psi}^{p, w}\right)^{*}=S_{\Psi}^{q, 1 / w}$, and $S_{\Psi}^{p, w}=\left.S_{\Psi}^{\infty, w}\right|_{\mathscr{H}_{w}^{p}}$. The operator $S_{\Psi}^{p, w}$ is bounded with bound $\left\|G_{\widetilde{\Psi}, \Psi}^{p, w}\right\|_{\ell_{w}^{p} \rightarrow \ell_{w}^{p}}\left\|G_{\Psi}^{p, w}\right\|_{\ell_{w}^{p} \rightarrow \ell_{w}^{p}}$. It is invertible with inverse $\left(S_{\Psi}^{p, w}\right)^{-1}=S_{\widetilde{\Psi}}^{p, w}=$ $\left.S_{\widetilde{\Psi}}^{\infty, w}\right|_{\mathscr{H}_{w}^{p}}=\left(\left.S_{\Psi}^{\infty, w}\right|_{\mathscr{H}_{w}^{p}}\right)^{-1}$, and is therefore simultaneously invertible on all $\mathscr{H}_{w}^{p}$. (iv) For the Gram matrix $\left(G_{\Psi}\right)_{k, l}=\left\langle\psi_{l}, \psi_{k}\right\rangle_{\mathscr{H}}$ (which by the admissibility induces a bounded operator $G_{\Psi}^{p, w}: \ell_{w}^{q} \rightarrow \ell_{w}^{p}$ ) we have $G_{\Psi}^{p, w}=C_{\Psi}^{p, w} D_{\Psi}^{p, w}$ and again $G_{\Psi}^{p, w}=$ $\left.G_{\Psi}^{\infty, w}\right|_{\mathscr{H}_{w}^{p}}$ The operator $G_{\Psi, \widetilde{\Psi}}$ is the projection from $\ell_{w}^{p}$ on $\operatorname{ran}\left(C_{\Psi}^{p, w}\right)$.

As a consequence of Theorem 4 , one may now drop the indices and write simply and unambiguously $C_{\Psi}, D_{\Psi}, S_{\Psi}$ and $G_{\Psi}$.

We split the proof of Theorem 4 into shorter lemmata. Note that we prove them for an arbitrary frame $\Phi$ that is localized with respect to the intrinsically localized frame $\Psi$. So we need the following preparatory result.

Lemma 5. Let $\Phi$ and $\Psi$ frames with $\Phi \sim_{\mathscr{A}} \Psi$ and $\Psi \sim_{\mathscr{A}} \Psi$ and let $w$ be an $\mathscr{A}$ admissible weight. Then $\Phi$ is intrinsically localized, and $\widetilde{\Phi} \sim_{\mathscr{A}} \widetilde{\Psi}$ and $\Phi \sim_{\mathscr{A}} \widetilde{\Psi}$.

In particular $\phi_{k} \in \mathscr{H}_{1 / w}^{1}$ and $\left\langle f, \phi_{k}\right\rangle_{\mathscr{H}_{w}^{p}, \mathscr{H}_{1 / w}^{q}}=\left\langle f, \phi_{k}\right\rangle_{\mathscr{H}_{w}^{\infty}, \mathscr{H}_{1 / w}^{1}}$ for $f \in \mathscr{H}_{w}^{p}$.

Proof. Since $\widetilde{\Psi} \sim \mathscr{A} \widetilde{\Psi}$ by Theorem 2 , we may apply (5) as follows:

$$
\begin{aligned}
& \Phi \sim_{\mathscr{A}} \Psi, \widetilde{\Psi} \sim_{\mathscr{A}} \widetilde{\Psi} \Rightarrow \Phi \sim_{\mathscr{A}} \widetilde{\Psi} \\
& \Phi \sim_{\mathscr{A}} \Psi, \widetilde{\Psi} \sim_{\mathscr{A}} \Phi \Rightarrow \Phi \sim_{\mathscr{A}} \Phi .
\end{aligned}
$$

As a consequence, the frame $\Phi$ is $\mathscr{A}$-localized and all results about $\mathscr{A}$-localized frames apply to $\Phi$. In particular, Proposition 1 implies that $\mathscr{H}_{w}^{p}(\Psi, \widetilde{\Psi})=$ $\mathscr{H}_{w}^{p}(\Phi, \widetilde{\Phi})$ with equivalent norms, and we may write unambiguously $\mathscr{H}_{w}^{p}$.

Furthermore, since the Gram matrix $G_{\widetilde{\Psi}, \Phi} \in \mathscr{A}$ is bounded on $\ell_{w}^{1}$, it follows that every row and column of $G_{\widetilde{\Psi}, \Phi}$ belongs to $\ell_{w}^{1}$ and likewise to $\ell_{1 / w}^{1}$. Consequently, $\phi_{k}=\sum_{l \in K}\left\langle\phi_{k}, \tilde{\psi}_{l}\right\rangle \psi_{l}$ is in $\mathscr{H}_{w}^{1} \cap \mathscr{H}_{1 / w}^{1}$. Thus the brackets

$$
\left\langle f, \phi_{k}\right\rangle_{\mathscr{H}_{w}^{p}, \mathscr{H}_{1 / w}^{q}}=\left\langle f, \phi_{k}\right\rangle_{\mathscr{H}_{w}^{\infty}, \mathscr{H}_{1 / w}^{1}}
$$

are well-defined by Lemma4

In particular all results shown above are applicable also for $\Phi$, however, with equivalent norms. 
Lemma 6. Let $\Phi$ and $\Psi$ be $\mathscr{A}$-localized frames such that $\Phi \sim_{\mathscr{A}} \Psi$. Let $w$ be an $\mathscr{A}$-admissible weight and $1 \leq p, q \leq \infty$ with $1 / p+1 / q=1$ or $(p, q)=(0,1)$. Then the analysis operator $C_{\Phi}^{p, w}: \mathscr{H}_{w}^{p} \rightarrow \ell_{w}^{p}$ given by

$$
C_{\Phi}^{p, w} f=\left(\left\langle f, \phi_{k}\right\rangle_{\mathscr{H}_{w}^{p}, \mathscr{H}_{1 / w}^{q}}, k \in K\right)
$$

is bounded, one-to-one and has closed range. Furthermore

$$
\frac{1}{\left\|G_{\widetilde{\Psi}, \widetilde{\Phi}}^{p, w}\right\|_{\ell_{w}^{p} \rightarrow \ell_{w}^{p}}}\|f\|_{\mathscr{H}_{w}^{p}} \leq\left\|C_{\Phi}^{p, w} f\right\|_{\ell_{w}^{p}} \leq\left\|G_{\Phi, \Psi}^{p, w}\right\|_{\ell_{w}^{p} \rightarrow \ell_{w}^{p}}\|f\|_{\mathscr{H}_{w}^{p}},
$$

where both sides of the inequality are bounded. The operator $C_{\Phi}^{p, w}$ is the restriction of $C_{\Phi}^{\infty, w}$ to $\mathscr{H}_{w}^{p}$, i.e. $C_{\Phi}^{p, w}=\left.C_{\Phi}^{\infty, w}\right|_{\mathscr{H}_{w}^{p}}$.

Proof. The associated Banach spaces $\mathscr{H}_{w}^{p}$ coincide for the frames $\Psi$ and $\Phi$. By Proposition11 and Lemma四 $\left(C_{\Phi}^{p, w} f\right)_{k}=\left\langle f, \phi_{k}\right\rangle_{\mathscr{H}_{w}^{p}, \mathscr{H}_{1 / w}^{q}}=\left\langle f, \phi_{k}\right\rangle_{\mathscr{H}_{w}^{\infty}, \mathscr{H}_{1 / w}^{1}}$.

$$
\begin{aligned}
& \left\|C_{\widetilde{\Psi}}^{p, w} f\right\|_{\ell_{w}^{p}}=\left\|C_{\widetilde{\Psi}}^{p, w} D_{\widetilde{\Phi}}^{p, w} C_{\Phi}^{p, w} f\right\|_{\ell_{w}^{p}} \leq\left\|G_{\widetilde{\Psi}, \widetilde{\Phi}}^{p, w}\right\|_{\ell_{w}^{p} \rightarrow \ell_{w}^{p}}\left\|C_{\Phi}^{p, w} f\right\|_{\ell_{w}^{p}} \text {, and } \\
& \left\|C_{\Phi}^{p, w} f\right\|_{\ell_{w}^{p}}=\left\|C_{\Phi}^{p, w} D_{\Psi}^{p, w} C_{\widetilde{\Psi}}^{p, w} f\right\|_{\ell_{w}^{p}} \leq\left\|G_{\Phi, \Psi}^{p, w}\right\|_{\ell_{w}^{p} \rightarrow \ell_{w}^{p}}\left\|C_{\widetilde{\Psi}}^{p, w} f\right\|_{\ell_{w}^{p}} .
\end{aligned}
$$

By Lemma 5 the Gram matrices $G_{\widetilde{\Psi}}^{p, w}$ and $G_{\Phi, \Psi}^{p, w}$ are in $\mathscr{A}$ and are therefore bounded on $\ell_{w}^{p}$ for all $p, 1 \leq p \leq \infty$. By Lemma $4 C_{\Psi}^{p, w}=C_{\Psi}^{\infty, w}{ }_{{ }_{\mathscr{H}_{w}^{p}}^{p}}$, since $\mathscr{H}_{w}^{p} \subseteq \mathscr{H}_{w}^{\infty}$.

As $\widetilde{\Psi} \sim_{\mathscr{A}} \Psi$ the analysis operator $C_{\widetilde{\Psi}}^{p, w}: \mathscr{H}_{w}^{p} \rightarrow \ell_{w}^{p}$ is given by $C_{\tilde{\Psi}}^{p, w} f=\left\langle f, \tilde{\psi}_{k}\right\rangle_{\mathscr{H}_{w}^{p}, \mathscr{H}_{1 / w}^{q}}$. By definition, this particular analysis operator is an isometry.

Lemma 7. Under the assumptions of Lemma 6 the synthesis operator $D_{\Phi}^{p, w}: \ell_{w}^{p} \rightarrow$ $\mathscr{H}_{w}^{p}$ is bounded and onto with operator norm

$$
\left\|D_{\Phi}^{p, w}\right\|_{\ell_{w}^{p} \rightarrow \mathscr{H}_{w}^{p}}=\left\|G_{\widetilde{\Psi}, \Phi}^{p, w}\right\|_{\ell_{w}^{p} \rightarrow \ell_{w}^{p}} .
$$

It is given by

$$
D_{\Phi}^{p, w} c=\sum_{k} c_{k} \phi_{k}
$$

with unconditional convergence in $\mathscr{H}_{w}^{p}$ for $1 \leq p<\infty$ and $p=0$ and weak $*_{-}$ convergence for $p=\infty$. Furthermore, $D_{\Phi}^{p, w}$ is the restriction of $D_{\Phi}^{\infty, w}$ to $\mathscr{H}_{w}^{p}$, i.e. $D_{\Phi}^{p, w}=\left.D_{\Phi}^{\infty, w}\right|_{\mathscr{H}_{w}^{p}} ^{p}$. For $p<\infty$ we have

$$
\left(D_{\Phi}^{p, w}\right)^{*}=C_{\Phi}^{q, 1 / w} \text { and } \quad\left(C_{\Phi}^{p, w}\right)^{*}=D_{\Phi}^{q, 1 / w}
$$


Proof. By Lemma 5 and Lemma $3 D_{\Phi}^{p, w}$ is bounded and onto $\mathscr{H}_{w}^{p}(\Phi, \widetilde{\Phi})=$ $\mathscr{H}_{w}^{p}(\Psi, \widetilde{\Psi})$ (see also Proposition 11). Since $D_{\Phi}^{p, w}=G_{\Phi, \widetilde{\Psi}} D_{\Psi}^{p, w}, D_{\Phi}^{p, w}$ is bounded on $\ell_{w}^{p}$. The unconditional convergence of $D_{\Phi}^{p, w} c=\sum_{k} c_{k} \phi_{k}$. is shown as in Lemma 2 Since $\ell_{w}^{p} \subset \ell_{w}^{\infty}$, it is clear that $D_{\Phi}^{p, w}=\left.D_{\Phi}^{\infty, w}\right|_{\mathscr{H}_{w}^{p}}$.

For the adjoint operator let $c \in \ell_{w}^{p}$, and $f \in \mathscr{H}_{1 / w}^{q} \simeq\left(\mathscr{H}_{w}^{p}\right)^{\prime}$. Then

$$
\begin{aligned}
& \left\langle D_{\Phi}^{p, w} c, f\right\rangle_{\mathscr{H}_{w}^{p}, \mathscr{H}_{1 / w}^{q}}=\left\langle\sum_{k} c_{k} \phi_{k}, f\right\rangle_{\mathscr{H}_{w}^{p}, \mathscr{H}_{1 / w}^{q}}= \\
& =\sum_{k} c_{k}\left\langle\phi_{k}, f\right\rangle_{\mathscr{H}_{w}^{p}, \mathscr{H}_{1 / w}^{q}}=\left\langle c, C_{\Phi}^{q, 1 / w} f\right\rangle_{\ell_{w}^{p}, \ell_{1 / w}^{q}},
\end{aligned}
$$

where the change of order is justified because $c \in \ell_{w}^{p}, f \in \mathscr{H}_{1 / w}^{q}$ and by Lemma 6

The operator norm is

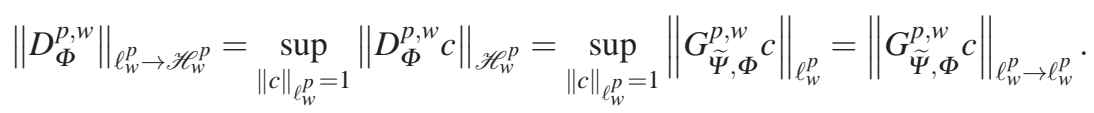

Clearly, by above, $\left\|D_{\Phi}^{p, w}\right\|_{\ell_{w}^{p} \rightarrow \mathscr{H}_{w}^{p}}=\left\|C_{\Phi}^{q, 1 / w}\right\|_{\left(\mathscr{H}_{w}^{p}\right)^{\prime} \rightarrow \ell_{1 / w}^{q}}$. In general we have $\left\|C_{\Phi}^{q, 1 / w}\right\|_{\left(\mathscr{H}_{w}^{p}\right)^{\prime} \rightarrow \ell_{1 / w}^{q}} \neq\left\|C_{\Phi}^{q, 1 / w}\right\|_{\mathscr{H}_{1 / w}^{q} \rightarrow \ell_{1 / w}^{q}}$, because the isomorphism between $\left(\mathscr{H}_{w}^{p}\right)^{\prime}$ and $\mathscr{H}_{1 / w}^{q}$ of Proposition 2 is not an isometry.

Lemma 8. Let $\Psi, \Phi$ and $\Xi$ be frames with $\Psi \sim_{\mathscr{A}} \Psi, \Phi \sim_{\mathscr{A}} \Psi$ and $\Xi \sim_{\mathscr{A}} \Psi$, and let $w$ be an $\mathscr{A}$-admissible weight. Let $1 \leq p \leq \infty$ and let $1 / p+1 / q=1$ or $(p, q)=(0,1)$. The cross-Gram matrix $G_{\Phi, \Xi}$ with entries $\left(G_{\Phi, \Xi}\right)_{k, l}=\left\langle\xi_{l}, \phi_{k}\right\rangle_{\mathscr{H}}$ induces a bounded operator $G_{\Phi, \Xi}^{p, w}: \ell_{w}^{p} \rightarrow \ell_{w}^{p}$ and factors as

$$
G_{\Phi, \Xi}^{p, w}=C_{\Phi}^{p, w} D_{\Xi}^{p, w}
$$

and so $\left(G_{\Phi, \Xi}^{p, w}\right)^{*}=G_{\Xi, \Phi}^{q, 1 / w}$. The Gram matrix $G_{\Phi, \Xi}^{p, w}$ is the restriction $\left.G_{\Phi, \Xi}^{\infty, w}\right|_{\mathscr{H}_{w}^{p}}$.

Furthermore $\operatorname{ran}\left(G_{\Phi, \Xi}^{p, w}\right)=\operatorname{ran}\left(C_{\Phi}^{p, w}\right)$ and $\operatorname{ker}\left(G_{\Phi, \Xi}^{p, w}\right)=\operatorname{ker}\left(D_{\Xi}^{p, w}\right)$. The Gram matrix $G_{\Phi, \Xi}^{p, w}$ is a bijective mapping from $\operatorname{ran}\left(C_{\widetilde{\Xi}}^{p, w}\right)$ onto $\operatorname{ran}\left(C_{\Phi}^{p, w}\right)$.

For $\Xi=\widetilde{\Phi}$ the Gram matrix satisfies

$$
G_{\Phi, \widetilde{\Phi}}=G_{\widetilde{\Phi}, \Phi}=G_{\Phi, \widetilde{\Phi}}^{*}
$$

and $G_{\Phi, \widetilde{\Phi}}$ is a bounded projection from $\ell_{w}^{p}$ on the range of $C_{\Phi}^{p, w}$ with kernel $\operatorname{ker}\left(D_{\Phi}^{p, w}\right)$. In addition, $\operatorname{ran}\left(C_{\Phi}^{p, w}\right)=\operatorname{ran}\left(C_{\widetilde{\Phi}}^{p, w}\right)$ and 


$$
\ell_{w}^{p}=\operatorname{ran}\left(C_{\Phi}^{p, w}\right) \oplus \operatorname{ker}\left(D_{\Phi}^{p, w}\right)
$$

Therefore, we have

$$
\left\|D_{\Phi}^{p, w}\right\|_{\ell_{w}^{p} \rightarrow \mathscr{H}_{w}^{p}}=\left\|G_{\widetilde{\Phi}, \Phi}\right\|_{\ell_{w}^{p} \rightarrow \ell_{w}^{p}} \geq 1
$$

Proof. For $c \in \ell^{00}$ we have

$$
\left(G_{\Phi, \Xi}^{p, w} \cdot c\right)_{l}=\sum_{k}\left(G_{\Phi, \Xi}\right)_{l, k} c_{k}=\sum_{k}\left\langle\xi_{k}, \phi_{l}\right\rangle c_{k}=\left(C_{\Phi} D_{\Xi} c\right)_{l}
$$

Therefore $G_{\Phi, \Xi}^{p, w}=C_{\Phi}^{p, w} D_{\Xi}^{p, w}$ on $\ell^{00}$. Since both sides are bounded operators on $\ell_{w}^{p}$ $\left(G_{\Phi, \Xi}^{p, w}\right.$ because $\left.\Phi \sim \mathscr{A} \Xi\right)$, the factorization can be extended from the dense subspace $\ell^{00}$ to $\ell_{w}^{p}$ for $p<\infty$.

By Lemma $6 C_{\Phi}^{p, w}$ is one-to-one, and therefore $\operatorname{ker}\left(G_{\Phi, \Xi}^{p, w}\right)=\operatorname{ker}\left(D_{\Xi}^{p, w}\right)$. Likewise, by Lemma $7 D_{\Xi}^{p, w}$ is onto $\mathscr{H}_{w}^{p}$, and therefore $\operatorname{ran}\left(G_{\Phi, \Xi}^{p, w}\right)=\operatorname{ran}\left(C_{\Phi}^{p, w}\right)$. Since $G_{\Phi, \Xi}^{p, w} C_{\widetilde{\Xi}}^{p, w} f=C_{\Phi}^{p, w} f$, the Gram matrix $G_{\Phi, \Xi}^{p, w}$ induces a bijective mapping from $\operatorname{ran}\left(C_{\Xi}^{\underline{\underline{p}, w}}\right)$ onto ran $\left(C_{\Phi}^{p, w}\right)$. (Compare to the 'frame transformation' in [4].)

If $\Xi=\widetilde{\Phi}$, then

$$
\left(G_{\Phi, \widetilde{\Phi}}\right)_{k, l}=\left\langle\phi_{l}, \tilde{\phi}_{k}\right\rangle_{\mathscr{H}}=\left\langle\phi_{l}, S^{-1} \phi_{k}\right\rangle_{\mathscr{H}}=\left\langle S^{-1} \phi_{l}, \phi_{k}\right\rangle_{\mathscr{H}}=\left(G_{\widetilde{\Phi}, \Phi}\right)_{k, l}
$$

and for the entries of the adjoint matrix

$$
\left(G_{\Phi, \widetilde{\Phi}}^{*}\right)_{k, l}={\overline{\left(G_{\Phi, \widetilde{\Phi}}\right)_{l, k}}}^{=} \overline{\left\langle\phi_{k}, S^{-1} \phi_{l}\right\rangle_{\mathscr{H}}}=\left\langle S^{-1} \phi_{l}, \phi_{k}\right\rangle_{\mathscr{H}}=\left(G_{\Phi, \widetilde{\Phi}}\right)_{k, l},
$$

and (14) is verified.

Since $D_{\tilde{\Phi}}^{p, w} C_{\Phi}^{p, w}=\operatorname{Id}_{\mathscr{H}_{w}^{p}}$ by $[6$, we obtain

$$
\left(G_{\Phi, \widetilde{\Phi}}^{p, w}\right)^{2}=C_{\Phi}^{p, w} D_{\tilde{\Phi}}^{p, w} C_{\Phi}^{p, w} D_{\tilde{\Phi}}^{p, w}=C_{\Phi}^{p, w} D_{\tilde{\Phi}}^{p, w}=G_{\Phi, \widetilde{\Phi}}^{p, w}
$$

Thus $G_{\Phi, \widetilde{\Phi}}^{p, w}$ is a projection operator on $\ell_{w}^{p}$ with range $\operatorname{ran}\left(C_{\Phi}^{p, w}\right)$ and kernel $\operatorname{ker}\left(D_{\tilde{\Phi}}^{p, w}\right)=\operatorname{ker}\left(D_{\Phi}^{p, w}\right)$. In particular, $\left\|G_{\Phi, \widetilde{\Phi}}^{p, w}\right\| \geq 1$ and 15 follows.

Since $G_{\Phi, \widetilde{\Phi}}^{p, w}=G_{\widetilde{\Phi}, \Phi}^{p, w}$ we get $\operatorname{ran}\left(G_{\Phi, \widetilde{\Phi}}^{p, w}\right)=\operatorname{ran}\left(G_{\widetilde{\Phi}, \Phi}^{p, w}\right)$ and by above we also have $\operatorname{ran}\left(C_{\Psi}^{p, w}\right)=\operatorname{ran}\left(C_{\widetilde{\Psi}}^{p, w}\right)$. By the projection property, using [18, Theorem III.13.2], $\operatorname{ran}\left(C_{\Phi}^{p, w}\right)$ and $\operatorname{ker}\left(D_{\Phi}^{p, w}\right)$ are therefore complementary subspaces.

By combining all properties of $C_{\Psi}^{p, w}$ and $D_{\Psi}^{p, w}$, we finally obtain the following list of properties for the frame operator $S_{\Phi}^{p, w}=D_{\Phi}^{p, w} C_{\Phi}^{p, w}$.

Lemma 9. The frame operator $S_{\Phi}^{p, w}: \mathscr{H}_{w}^{p} \rightarrow \mathscr{H}_{w}^{p}$ is defined by 


$$
S_{\Phi}^{p, w} f=\sum_{k}\left\langle f, \phi_{k}\right\rangle_{\mathscr{H}_{w}^{p}, \mathscr{H}_{1 / w}^{q}} \cdot \phi_{k}=\sum_{k}\left\langle f, \phi_{k}\right\rangle_{\mathscr{H}_{w}^{\infty}, \mathscr{H}_{1 / w}^{1}} \cdot \phi_{k}
$$

with unconditional convergence in $\mathscr{H}_{w}^{p}$ for $p<\infty$ and weak ${ }^{*}$-unconditional convergence for $p=\infty$. The frame operator satisfies the identities $S_{\Phi}^{p, w}=D_{\Phi}^{p, w} C_{\Phi}^{p, w}$, $\left(S_{\Phi}^{p, w}\right)^{*}=S_{\Phi}^{q, 1 / w}$, and $S_{\Phi}^{p, w}=\left.S_{\Phi}^{\infty, w}\right|_{\mathscr{H}} ^{p}$, and is bounded on all $\mathscr{H}^{p, w}$ with operator norm

$$
\left\|S_{\Phi}^{p, w}\right\|_{\mathscr{H}_{w}^{p} \rightarrow \mathscr{H}_{w}^{p}} \leq\left\|G_{\widetilde{\Psi}, \Phi}^{p, w}\right\|_{\ell_{w}^{p} \rightarrow \ell_{w}^{p}} \cdot\left\|G_{\Phi, \Psi}^{p, w}\right\|_{\ell_{w}^{p} \rightarrow \ell_{w}^{p}} \cdot
$$

Furthermore, $S_{\Phi}^{p, w}$ is simultaneously invertible on all $\mathscr{H}_{w}^{p}$ with inverse $\left(S_{\Phi}^{p, w}\right)^{-1}=$ $S_{\widetilde{\Phi}}^{p, w}$.

\section{Galerkin Matrix representation of operators with localized frames}

For a numerical treatment of operator equations one often uses redundant frame representations for the Galerkin discretization. Such discretizations have been formulated for wavelet frames in [75] and for Gabor frames in [51]. The formalism for general (Hilbert space) frames has been introduced in [5].

For localized frames we formally define the relation between operators and matrices as follows.

Definition 4. Let $\Psi, \Phi$ and $\Xi$ be frames with $\Psi \sim_{\mathscr{A}} \Psi, \Phi \sim_{\mathscr{A}} \Psi$ and $\Xi \sim_{\mathscr{A}} \Psi$. Let $w_{1}, w_{2}$ be $\mathscr{A}$-admissible weights and $1 \leq p_{1}, p_{2} \leq \infty$ or $p_{1}, p_{2}=0$. Let $q_{1}, q_{2}$ be the dual indices defined as usual.

(i) For the bounded linear operator $O: \mathscr{H}_{w_{1}}^{p_{1}} \rightarrow \mathscr{H}_{w_{2}}^{p_{2}}$ define the matrix $\mathscr{M}_{(\Phi, \Xi)}$ by

$$
\left(\mathscr{M}_{(\Phi, \Xi)}(O)\right)_{k, l}=\left\langle O \xi_{l}, \phi_{k}\right\rangle_{\mathscr{H}_{w_{2}}^{p_{2}}, \mathscr{H}_{1 / w_{2}}^{q_{2}}}=\left\langle O \xi_{l}, \phi_{k}\right\rangle_{\mathscr{H}_{w_{2}}^{\infty}, \mathscr{H}_{1 / w_{2}}^{1}}
$$

We call $\mathscr{M}_{(\Phi, \Xi)}(O)$ the (Galerkin) matrix of $O$ with respect to $\Phi$ and $\Xi$.

(ii) For the matrix $M$ that induces a bounded operator in $\mathfrak{B}\left(\ell_{w_{1}}^{p_{1}}, \ell_{w_{2}}^{p_{2}}\right)$ define $\mathscr{O}_{(\Phi, \Xi)}$ : $\mathfrak{B}\left(\ell_{w_{1}}^{p_{1}}, \ell_{w_{2}}^{p_{2}}\right) \rightarrow \mathfrak{B}\left(\mathscr{H}_{w_{1}}^{p_{1}}, \mathscr{H}_{w_{2}}^{p_{2}}\right)$ by

$$
\left(\mathscr{O}_{(\Phi, \Xi)}(M)\right) h=\sum_{k}\left(\sum_{j} M_{k, j}\left\langle h, \xi_{j}\right\rangle\right) \phi_{k}
$$

for $h \in \mathscr{H}_{w_{1}}^{p_{1}}$. We call $\mathscr{O}_{(\Phi, \Xi)}(M)$ the operator of $M$ with respect to $\Phi$ and $\Xi$.

Theorem 5. Assume that $\Phi, \Psi$ and $\Xi$ are $\mathscr{A}$-localized frames in $\mathscr{H}$ satisfying $\Phi \sim_{\mathscr{A}} \Psi$ and $\Xi \sim_{\mathscr{A}} \Psi$. Let $w_{1}, w_{2}$ be $\mathscr{A}$-admissible weights, let $1 \leq p_{1}, p_{2} \leq \infty$ or $p_{1}, p_{2}=0$ with dual indices $q_{1}, q_{2}$.

(i) If $O \in \mathfrak{B}\left(\mathscr{H}_{w_{1}}^{p_{1}}, \mathscr{H}_{w_{2}}^{p_{2}}\right)$, then $\mathscr{M}_{(\Phi, \Xi)}(O) \in \mathfrak{B}\left(\ell_{w_{1}}^{p_{1}}, \ell_{w_{2}}^{p_{2}}\right)$, and we have 


$$
\left\|\mathscr{M}_{(\Phi, \Xi)}(O)\right\|_{\ell_{w_{1}}^{p_{1}} \rightarrow \ell_{w_{2}}^{p_{2}}} \leq\left\|G_{\Phi, \Psi}\right\|_{\ell_{w_{2}}^{p_{2}} \rightarrow \ell_{w_{2}}^{p_{2}}}\left\|G_{\widetilde{\Psi}, \Xi}\right\|_{\ell_{w_{1}}^{p_{1}} \rightarrow \ell_{w_{1}}^{p_{1}}}\|O\|_{\mathscr{H}_{w_{1}}^{p_{1} \rightarrow \mathscr{H}_{w_{2}}^{p_{2}}}}
$$

Furthermore,

$$
\mathscr{M}_{(\Phi, \Xi)}(O)=C_{\Phi} \circ O \circ D_{\Xi} .
$$

(ii) If $M \in \mathfrak{B}\left(\ell_{w_{1}}^{p_{1}}, \ell_{w_{2}}^{p_{2}}\right)$, then $\mathscr{O}_{(\Phi, \Xi)}(M) \in \mathfrak{B}\left(\mathscr{H}_{w_{1}}^{p_{1}}, \mathscr{H}_{w_{2}}^{p_{2}}\right)$, and

$$
\mathscr{O}_{(\Phi, \Xi)}(M)=D_{\Phi} \circ M \circ C_{\Xi},
$$

and

$$
\left\|\mathscr{O}_{(\Phi, \Xi)}(M)\right\|_{\mathscr{H}_{w_{2}}^{p_{2}} \rightarrow \mathscr{H}_{w_{1}}^{p_{1}}} \leq\left\|G_{\widetilde{\Psi}, \Phi}\right\|_{\ell_{w_{1}}^{p_{1}} \rightarrow \ell_{w_{1}}^{p_{1}}}\left\|G_{\Xi, \Psi}\right\|_{\ell_{w_{2}}^{p_{2}} \rightarrow \ell_{w_{2}}^{p_{2}}}\|M\|_{\ell_{w_{2}}^{p_{2}} \rightarrow \ell_{w_{1}}^{p_{1}}}
$$

Proof. This result follows directly from the results in Section 3 . For example, let $c=\left(c_{k}\right) \in \ell_{w_{1}}^{p_{1}}$, then

$$
\begin{gathered}
\left(\mathscr{M}_{(\Phi, \Xi)}(O) c\right)_{l}=\left(C_{\Phi} \circ O \circ D_{\Xi} c\right)_{l}= \\
=\left(C_{\Phi}\left(\sum_{k \in K} c_{k} O \xi_{k}\right)\right)_{l}=\sum_{k \in K} c_{k}\left\langle O \xi_{k}, \phi_{l}\right\rangle_{\mathscr{H}_{w_{2}}^{p_{2}}, \mathscr{H}_{1 / w_{2}}^{q_{2}}} .
\end{gathered}
$$

Using tensor products and the results in Section 3 it is easy to extend the results in [5]. For $g \in X_{1}^{\prime}, f \in X_{2}$ the tensor product $f \otimes g$ is defined as the rankone operator from $X_{1}$ to $X_{2}$ by $(f \otimes g)(h)=\langle h, g\rangle_{X_{1}, X_{1}^{\prime}} f$. We will use $(f \otimes g)(h)=$ $\langle h, g\rangle_{\mathscr{H}_{w}^{p}, \mathscr{H}_{1 / w}^{q}} f$ for $h \in \mathscr{H}_{w}^{p}$.

Proposition 4. Let $\Psi$ and $\Phi$ be $\mathscr{A}$-localized frames in $\mathscr{H}$ satisfying $\Phi \sim_{\mathscr{A}} \Psi$ and $w_{1}, w_{2}$ be $\mathscr{A}$-admissible weights and $1 \leq p_{1}, p_{2} \leq \infty$ or $p_{1}, p_{2}=0$. Then the factorization

$$
\left(\mathscr{O}_{(\Phi, \Psi)} \circ \mathscr{M}_{(\widetilde{\Phi}, \widetilde{\Psi})}\right)=\mathrm{id}=\left(\mathscr{O}_{(\widetilde{\Phi}, \widetilde{\Psi})} \circ \mathscr{M}_{(\Phi, \Psi)}\right)
$$

holds for every space of bounded operators $\mathfrak{B}\left(\mathscr{H}_{w_{1}}^{p_{1}}, \mathscr{H}_{w_{2}}^{p_{2}}\right)$.

Therefore every $O \in \mathfrak{B}\left(\mathscr{H}_{w_{1}}^{p_{1}}, \mathscr{H}_{w_{2}}^{p_{2}}\right)$ possesses the representation

$$
O=\sum_{k, j}\left\langle O \tilde{\psi}_{j}, \tilde{\phi}_{k}\right\rangle \phi_{k} \otimes \psi_{j}=\sum_{k, j}\left\langle O \psi_{j}, \phi_{k}\right\rangle \tilde{\phi}_{k} \otimes \tilde{\psi}_{j}
$$

and both expansions converge unconditionally in the strong operator topology (respectively weak-* unconditionally if either $p_{1}=\infty$ or $p_{2}=\infty$ ).

Proof. By Theorem 5 for an $O$ in any $\mathfrak{B}\left(\mathscr{H}_{w_{1}}^{p_{1}}, \mathscr{H}_{w_{2}}^{p_{2}}\right)$ we have

$$
\left(\mathscr{O}_{(\Phi, \Psi)} \circ \mathscr{M}_{(\widetilde{\Phi}, \widetilde{\Psi})}\right) O=D_{\Phi}\left(C_{\widetilde{\Phi}} O D_{\widetilde{\Psi}}\right) C_{\Psi}=O
$$

using the reconstruction formulas in Theorem 2 and Lemma 3 . 

5

The representation in 197) converges in the strong operator topology by Theorem

As in the Hilbert space setting [5] we get the following decomposition.

Proposition 5. Let $\Psi, \Phi$ and $\Xi$ be $\mathscr{A}$-localized frames in $\mathscr{H}$ satisfying $\Phi \sim \mathscr{A}$ $\Psi, \Xi \sim \mathscr{A} \Psi$. Let $w_{1}, w_{2}, w_{3}$ be $\mathscr{A}$-admissible weights and $1 \leq p_{1}, p_{2} \leq \infty$ or $p_{1}, p_{2}=0$. Then for $O_{1}: \mathscr{H}_{w_{1}}^{p_{1}} \rightarrow \mathscr{H}_{w_{2}}^{p_{2}}$ and $O_{2}: \mathscr{H}_{w_{3}}^{p_{3}} \rightarrow \mathscr{H}_{w_{1}}^{p_{1}}$, we have

$$
\mathscr{M}_{(\Phi, \Psi)}\left(O_{1} \circ O_{2}\right)=\mathscr{M}_{(\Phi, \Xi)}\left(O_{1}\right) \circ \mathscr{M}_{(\widetilde{\Xi}, \Psi)}\left(O_{2}\right) .
$$

Proof. The statement follows from the factorization

$$
\begin{gathered}
\mathscr{M}_{(\Phi, \Psi)}\left(O_{1} \circ O_{2}\right)=C_{\Phi} O_{1} O_{2} D_{\Psi}= \\
=C_{\Phi} O_{1} D_{\widetilde{\Xi}} C_{\widetilde{\Xi}} O_{2} D_{\Psi}=\mathscr{M}_{(\Phi, \Xi)}\left(O_{1}\right) \circ \mathscr{M}_{(\widetilde{\Xi}, \Psi)}\left(O_{2}\right) .
\end{gathered}
$$

Then we get an extension of results in [7, 6] to coorbit spaces.

Lemma 10. Let $\Psi$, and $\Phi$ be $\mathscr{A}$-localized sequence in $\mathscr{H}$ satisfying $\Phi \sim \mathscr{A} \Psi$, $w_{1}, w_{2}$ be $\mathscr{A}$-admissible weights and $1 \leq p_{1}, p_{2} \leq \infty$ or $p_{1}, p_{2}=0$. Let $O$ be a linear operator from $\mathscr{H}^{00}$ into $\mathscr{H}_{w_{2}}^{\infty}$. Then

$$
O \in \mathfrak{B}\left(\mathscr{H}_{w_{1}}^{p_{1}}, \mathscr{H}_{w_{2}}^{p_{2}}\right) \Longleftrightarrow \mathscr{M}_{(\Psi, \Phi)}(O) \in \mathfrak{B}\left(\ell_{w_{1}}^{p_{1}}, \ell_{w_{2}}^{p_{2}}\right),
$$

Proof. The implication $\Rightarrow$ is stated in Theorem 5 (i).

For the converse, let $O$ be a linear operator from $\mathscr{H}^{00}$ to $\mathscr{H}_{w_{2}}^{p_{2}}$ such that $\mathscr{M}_{(\Psi, \Phi)}(O)=C_{\Phi}^{p_{2}, w_{2}} \circ O \circ D_{\Psi}^{p_{1}, w_{1}}$ is bounded. Then $\operatorname{ran}\left(D_{\Psi}\right) \subseteq \operatorname{dom}(O)$ and therefore $O$ is defined everywhere. Since

$$
O=D_{\widetilde{\Phi}}^{p_{2}, w_{2}} \circ C_{\Phi}^{p_{2}, w_{2}} \circ O \circ D_{\Psi}^{p_{1}, w_{1}} \circ C_{\widetilde{\Psi}}^{p_{1}, w_{1}},
$$

the operator $O$ is also bounded.

\subsection{Characterization of operator classes}

Combining the matrix representation of an operator with the well-known characterizations of boundedness of operators between $\ell^{p}$-spaces [67] we obtain criteria for the boundedness of operators between certain coorbit spaces.

For the description we recall the following norms on infinite matrices. Considering an index set $K=L \times N$, we can define discrete mixed norm spaces [47], i.e.,

$$
\ell_{w}^{p_{1}, p_{2}}=\left\{M=\left(M_{l, n}\right)_{l \in L, n \in N} \mid\|M\|_{\ell_{w}^{p_{1}, p_{2}}}:=\left(\sum_{l}\left(w_{l, n}\left|M_{l, n}\right|^{p_{2}}\right)^{p_{1} / p_{2}}\right)^{1 / p_{2}}<\infty\right\} .
$$


In particular we consider weights $w=w^{(1)} \otimes w^{(2)}$ with $w_{k, l}=w_{k}^{(1)} \cdot w_{l}^{(2)}$.

Proposition 6. Let $\Psi$ and $\Phi$ be $\mathscr{A}$-localized sequence in $\mathscr{H}$ satisfying $\Phi \sim \mathscr{A} \Psi$, $w^{(i)}$ be $\mathscr{A}$-admissible weights and $1 \leq p_{i}<\infty$ for $i=1,2$. Let $O$ be a linear operator from $\mathscr{H}^{00}$ to $\mathscr{H}_{w_{2}}^{\infty}$, and $M=\mathscr{M}_{(\Phi, \Xi)}(O)$. Then

$$
\begin{aligned}
& O \in \mathfrak{B}\left(\mathscr{H}_{w^{(1)}}^{\infty}, \mathscr{H}_{w^{(2)}}^{\infty}\right) \Longleftrightarrow O \in \mathfrak{B}\left(\mathscr{H}_{w^{(1)}}^{0}, \mathscr{H}_{w^{(2)}}^{\infty}\right) \\
& \Longleftrightarrow \sup _{k} \sum_{l}\left|w_{k}^{(2)} \cdot \frac{1}{w_{l}^{(1)}}\left\langle O \psi_{l}, \phi_{k}\right\rangle\right|<\infty \\
& \Longleftrightarrow M \in \ell_{1 / w^{(2)} \otimes w^{(1)}}^{\infty, 1} \text {. } \\
& O \in \mathfrak{B}\left(\mathscr{H}_{w^{(1)}}^{\infty}, \mathscr{H}_{w^{(2)}}^{0}\right) \Longleftrightarrow \lim _{k} \sum_{l}\left|w_{k}^{(2)} \cdot \frac{1}{w_{l}^{(1)}}\left\langle O \psi_{l}, \phi_{k}\right\rangle\right|=0 . \\
& O \in \mathfrak{B}\left(\mathscr{H}_{w^{(1)}}^{1}, \mathscr{H}_{w^{(2)}}^{\infty}\right) \Longleftrightarrow \sup _{k, l}\left|w_{k}^{(2)} \cdot\left\langle O \psi_{l}, \phi_{k}\right\rangle \cdot \frac{1}{w_{l}^{(1)}}\right|<\infty, \\
& \Longleftrightarrow M \in \ell_{1 / w^{(2)} \otimes w^{(1)}}^{\infty, \infty} \\
& O \in \mathfrak{B}\left(\mathscr{H}_{w^{(1)}}^{1}, \mathscr{H}_{w^{(2)}}^{p}\right) \Longleftrightarrow \sup _{l} \sum_{k}\left|w_{k}^{(2)} \cdot \frac{1}{w_{l}^{(1)}}\left\langle O \psi_{l}, \phi_{k}\right\rangle\right|^{p}<\infty, \\
& \Longleftrightarrow M^{*} \in \ell_{1 / w^{(2)} \otimes w^{(1)}}^{p, \infty} \\
& O \in \mathfrak{B}\left(\mathscr{H}_{w^{(1)}}^{\infty}, \mathscr{H}_{w^{(2)}}^{1}\right) \Longleftrightarrow \sup _{E \text { finite }} \sum_{l}\left|\sum_{k \in E} w_{k}^{(2)} \cdot \frac{1}{w_{l}^{(1)}}\left\langle O \psi_{l}, \phi_{k}\right\rangle\right|<\infty . \\
& O \in \mathfrak{B}\left(\mathscr{H}_{w^{(1)}}^{2}, \mathscr{H}_{w^{(2)}}^{2}\right) \Longleftrightarrow\left\{\begin{array}{c}
\text { For } \breve{M}_{k, l}=w_{k}^{(2)} \cdot \frac{1}{w_{l}^{(1)}}\left\langle\text { O } \psi_{l}, \phi_{k}\right\rangle \text { we have: } \\
\sum_{l}\left|\sum_{k \in E} \breve{M}_{k, l}\right|^{2}<\infty, \\
\left(\breve{M}^{*} \breve{M}\right)^{n} \text { is defined for all } n=1,2, \ldots \\
\sup _{n} \sup _{i}\left[\left(\left(\breve{M}^{*} \breve{M}\right)^{n}\right)_{i, i}\right]^{1 / n}=K<\infty .
\end{array}\right.
\end{aligned}
$$

Proof. The conditions on the matrix $M$ are variations of the well-known Schur test. For instance, $M$ is bounded from $\ell^{\infty}$ to $\ell^{\infty}$, if and only if the row sums are uniformly bounded, i.e., $\sup _{k} \sum_{l}\left|M_{k, l}\right|<\infty$. A convenient reference for Schur's test is [67.

To also include weights, we proceed as follows. Let $D_{j} c=\left(w_{k}^{(j)} c_{k}\right)_{k \in K}$ be the multiplication operator with weight $w^{(j)}, j=1,2$. Then $D_{j}$ is an isometric isomorphism from $\ell_{w^{(j)}}^{p}$ onto $\ell^{p}$.

Therefore a matrix $M$ is bounded from $\ell_{w^{(1)}}^{p_{1}}$ into $\ell_{w^{(2)}}^{p_{2}}$, if and only if $\breve{M}=$ $D_{2} M D_{1}^{-1}$ is bounded from $\ell^{p_{1}}$ into $\ell^{p_{2}}$. We now apply the boundedness characterizations in [67] to $D_{2} \mathscr{M}_{(\Psi, \Phi)}(O) D_{1}^{-1}$. For example, Lemma 10 says that $O \in$ $\mathfrak{B}\left(\mathscr{H}_{w_{1}}^{\infty}, \mathscr{H}_{w_{2}}^{\infty}\right) \Longleftrightarrow \mathscr{M}_{(\Psi, \Phi)}(O) \in \mathfrak{B}\left(\ell_{w_{1}}^{\infty}, \ell_{w_{2}}^{\infty}\right)$, which in turn is equivalent to saying 
that $\breve{M}=D_{2} \mathscr{M}_{(\Psi, \Phi)}(O) D_{1}^{-1} \in \mathfrak{B}\left(\ell^{\infty}, \ell^{\infty}\right)$. Since $\breve{M}_{k, l}=w_{k}^{(2)} \cdot \frac{1}{w_{l}^{(1)}}\left\langle O \psi_{l}, \phi_{k}\right\rangle$, condition (21) follows from cite[Theorem 2.6]Maddox:101881. The other characterization follow in the same way from [67, Theorem 2.12,2.13(a),2.13(b),2.14] and [25], respectively.

In concrete applications one uses only the sufficient conditions for boundedness and checks that the matrix $\mathscr{M}_{(\Psi, \Phi)}(O)$ satisfies the conditions of Schur's test.

Corollary 3. Let $\Psi$ and $\Phi$ be $\mathscr{A}$-localized frame in $\mathscr{H}$ satisfying $\Phi \sim \mathscr{A} \Psi$, and $1 \leq p \leq \infty$. Let $O$ be a linear operator from $\mathscr{H}^{00}$ to $\mathscr{H}_{w}^{\infty}$. If $\sup _{k} \sum_{l}\left|\left\langle O \psi_{l}, \phi_{k}\right\rangle\right|<\infty$, and $\sup _{l} \sum_{k}\left|\left\langle O \psi_{l}, \phi_{k}\right\rangle\right|<\infty$, then $O \in \mathfrak{B}\left(\mathscr{H}^{p}, \mathscr{H}^{p}\right)$.

For an example of how these abstract results are applied in analysis, we refer to the investigation of the boundedness of pseudodifferential operators with the help of Gabor frames in [51, 57].

For further reference, we remark that the results in Section 4 do not use the full power of intrinsic localization, but remain true under weaker assumptions. In fact, we have only used the norm equivalences for the analysis operators of two frames $\Phi$ and $\Psi$ and their duals $\tilde{\Phi}$ and $\tilde{\Psi}$ :

$$
\left\|C_{\Psi} f\right\|_{\ell_{w}^{p}} \asymp\left\|C_{\tilde{\Psi}} f\right\|_{\ell_{w}^{p}} \asymp\left\|C_{\Phi} f\right\|_{\ell_{w}^{p}} \asymp\left\|C_{\tilde{\Phi}} f\right\|_{\ell_{w}^{p}}
$$

for $f \in \mathscr{H}^{00}$. This is all that is needed to define unambiguously a coorbit space $\mathscr{H}_{w}^{p}$.

If (27) holds, then all statements of this section, specifically Theorem 5 and Propositions 4-6remain true.

One of our main points is that these norm equivalences 27) always hold for $\mathscr{A}$-localized frames, as we have seen in Section 3 In addition, (27) also hold for wavelet frames (with sufficiently many vanishing moments and sufficient decay) with the Besov spaces and Sobolev spaces as the corresponding coorbit spaces. In fact, one of the main motivations for wavelets was the investigation of singular integral operators, see [40, 69]. However, for wavelet frames the norm equivalences (27) require different arguments that are not covered by our theory of localized frames.

\subsection{Invertibility}

For the invertibility we can show, as in the Hilbert space setting [6]:

Lemma 11. Let $\Phi$ and $\Psi$ be $\mathscr{A}$-localized frames for $\mathscr{H}$ satisfying $\Phi \sim_{\mathscr{A}} \Psi$ and $1 \leq p \leq \infty$. Let $O: \mathscr{H}_{w_{1}}^{p} \rightarrow \mathscr{H}_{w_{2}}^{p}$ be a bounded, linear operator.

Then $O$ is bijective, if and only if $\mathscr{M}_{(\Phi, \Psi)}(O)$ is bijective as operator from $\operatorname{ran}\left(C_{\Psi}^{p, w_{1}}\right)$ to $\operatorname{ran}\left(C_{\Phi}^{p, w_{2}}\right)$.

In this case the matrix associated to the inverse is given by 


$$
\left(\mathscr{M}_{(\Phi, \Psi)}(O)\right)^{\dagger}:=\left(\mathscr{M}_{(\Phi, \Psi)}(O)_{\left.\right|_{\operatorname{ran}\left(C_{\Psi}\right)}}\right)^{-1}=\mathscr{M}_{(\widetilde{\Psi}, \widetilde{\Phi})}\left(O^{-1}\right) .
$$

Proof. By Theorem 4 $C_{\Phi}^{p, w}$ is a bijection from $\mathscr{H}_{w}^{p}$ onto $\operatorname{ran}\left(C_{\Phi}^{p, w}\right)$, and $D_{\Psi}^{p, w}$ a bijection from ran $\left(C_{\Psi}^{p, w}\right)$ onto $\mathscr{H}_{w}^{p}$, where $w=w_{1}$ or $w_{2}$.

Therefore $O$ is bijective if and only if $\mathscr{M}_{(\Phi, \Psi)}(O)$ is bijective from $\operatorname{ran}\left(C_{\Psi}^{p_{1}, w_{1}}\right)$ to $\operatorname{ran}\left(C_{\Psi}^{p_{2}, w_{2}}\right)$.

Furthermore

$$
\begin{gathered}
\mathscr{M}_{(\widetilde{\Psi}, \widetilde{\Phi})}\left(O^{-1}\right) \mathscr{M}_{(\Phi, \Psi)}(O)=C_{\widetilde{\Psi}}^{p_{1}, w_{1}} \circ O^{-1} \circ D_{\widetilde{\Phi}}^{p_{2}, w_{2}} C_{\Phi}^{p_{2}, w_{2}} \circ O \circ D_{\Psi}^{p_{1}, w_{1}}= \\
=C_{\widetilde{\Psi}}^{p_{1}, w_{1}} \circ D_{\Psi}^{p_{1}, w_{1}}=G_{\widetilde{\Psi}, \Psi},
\end{gathered}
$$

and therefore the projection on $\operatorname{ran}\left(C_{\Psi}^{p_{1}, w_{1}}\right)$.

Remark 2. Note that for $p_{1} \neq p_{2}$, there does not exist a bijective operator $O: \mathscr{H}_{w_{1}}^{p_{1}} \rightarrow$ $\mathscr{H}_{w_{2}}^{p_{2}}$ by Theorem 3 .

The condition number of a matrix (or an operator) plays an important role in numerical analysis [66] and is defined by $\kappa(M)=\|M\|_{O p} \cdot\left\|M^{-1}\right\|_{o p}$. For matrices with non-zero kernel we can define the generalized condition number [14] by $\kappa^{\dagger}(M)=\|M\|_{O p} \cdot\left\|M^{\dagger}\right\|_{o p}$. By using Lemma11 and Theorem5 it is straightforward to show

$$
\kappa^{\dagger}\left(\mathscr{M}_{(\Phi, \Psi)}(O)\right)=\kappa^{\dagger}\left(G_{\Phi, \Psi}\right) \cdot \kappa^{\dagger}\left(G_{\widetilde{\Psi}, \Psi}\right) \cdot \kappa^{\dagger}(O) .
$$

Theorem 6. Let $\Psi$ be an $\mathscr{A}$-localized frame for $\mathscr{H}$ and $w$ an $\mathscr{A}$-admissible weight. Assume that $O: \mathscr{H} \rightarrow \mathscr{H}$ is invertible and that $\mathscr{M}_{(\Psi, \tilde{\Psi})}(O) \in \mathscr{A}$. Then $O$ is invertible simultaneously on all coorbit spaces $\mathscr{H}_{w}^{p}, 1 \leq p \leq \infty$.

Proof. By Lemma 11 the matrix of $O^{-1}$ is given by $\mathscr{M}_{(\widetilde{\Psi}, \Psi)}\left(O^{-1}\right)=$ $\left(\mathscr{M}_{(\Psi, \tilde{\Psi})}(O)\right)^{\dagger}$. Since $\mathscr{A}$ is closed with respect to taking a pseudo-inverse and $\mathscr{M}_{(\Psi, \tilde{\Psi})}(O) \in \mathscr{A}$, it follows that also $\mathscr{M}_{(\widetilde{\Psi}, \Psi)}\left(O^{-1}\right) \in \mathscr{A} \subseteq \mathfrak{B}\left(\ell_{w}^{p}, \ell_{w}^{p}\right)$. By Lemma $10 O^{-1}$ is therefore bounded on $\mathscr{H}_{w}^{p}$ for $1 \leq p \leq \infty$.

This result is special for intrinsically localized frames and fails for wavelet frames.

\section{Outlook}

This manuscript was motivated by many discussions of the first author with applied scientists who work on the numerical solution of integral equations. In applications in acoustics, the solutions of the Helmholtz equation are of particular importance, see e.g. [61], they are used for example for the numerical estimation of head-related transfer functions [64, 79]. 


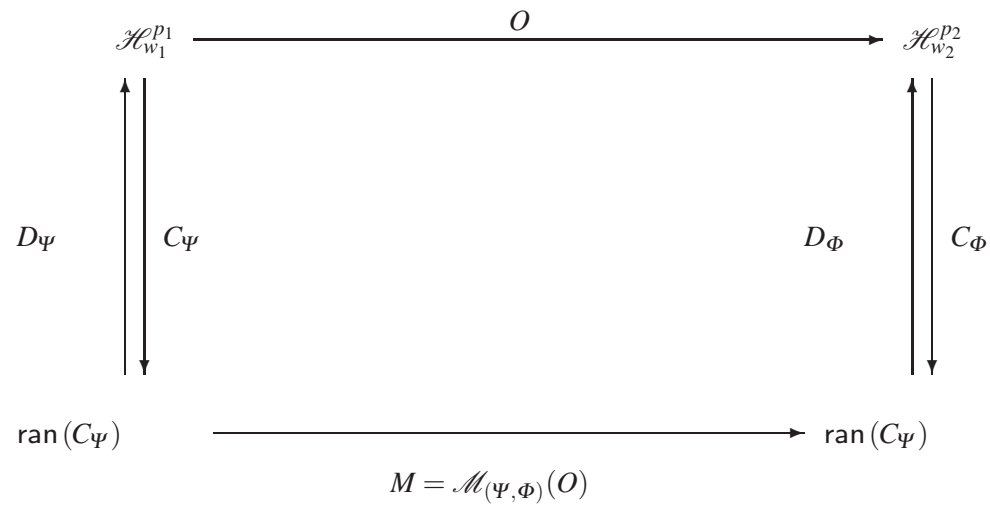

Fig. 1 All operators in the diagram are bijective, if $M$ or equivalently $O$, is bijective.

In general the problem of solving an integral equation can be seen as solving a linear equation

$$
O \cdot f=g,
$$

where the operator $O$ models the physical system and the right-hand side is given and the solution $f$ is to be determined. For the important example of sound propagation, the right-hand side $g$ is often called the load vector. It is usually assumed that $f, g$, are in some appropriate function spaces.

For the numerical treatment of such operator equations one needs a reduction to a discrete formulation. This is often done with a so-called Galerkin scheme [73]. As a first step, either the boundary of the considered space or the whole space itself are separated in finite patches or finite volume elements. This leads to the Boundary Element Method [43] or the Finite Element Method [9]. In the Galerkin scheme one first finds the matrix $M=\mathscr{M}_{(\Psi, \Psi)}(0)$ corresponding to the operator $O$ with respect to a given basis or frame $\Psi$. Instead of solving the operator equation $O f=g$, one converts (28) into a matrix equation as follows.

$$
\begin{gathered}
O f=g \Longleftrightarrow \sum_{l}\left\langle f, \tilde{\phi}_{l}\right\rangle O \phi_{l}=g \Longleftrightarrow \sum_{l}\left\langle f, \tilde{\phi}_{l}\right\rangle\left\langle O \phi_{l}, \phi_{k}\right\rangle=\left\langle g, \phi_{k}\right\rangle \Longleftrightarrow \\
\mathscr{M}_{(\Phi, \Phi)}(O) \cdot C_{\widetilde{\Phi}} f=C_{\Phi} g .
\end{gathered}
$$

Here $M=\mathscr{M}^{(\Phi, \tilde{\Phi})}(O)$ is called the system matrix or stiffness matrix.

In the setting of localized frames, the natural function spaces are the Banach spaces $\mathscr{H}_{w}^{p}$. By the results above this is equivalent to the vectors in the matrix equation (29) to be contained in some $\ell_{w}^{p}$-space.

In finite and boundary element approach the system $\Phi$ is usually a spline-like basis [43]. Recently wavelet bases [29], but also frames have been applied, e.g. in [75, 60]. Currently the potential of other frames is investigated for solving opera- 
tor equations in acoustics, such as $\alpha$-modulation frames [74]. Note, however, that neither wavelet frames nor $\alpha$-modulation frames are localized in the sense of Definition 11. As mentioned above, as long as (27) is fulfilled most results of Section 4 can still be applied.

To use a numerical solver, it is necessary to perform a further reduction to a finite-dimensional matrix equation. This means that we have to find a good finite dimensional approximation of $\mathscr{M}_{(\Phi, \Phi)}(O)$. This is done by restricting $\mathscr{M}_{(\Phi, \Phi)}(O)$ to a suitable finite-dimensional subspace. Specifically, let $\left\{P_{n}\right\}$ be a bounded sequence of finite-rank orthogonal projections in $\mathfrak{B}(\mathscr{H})$ with the property that $P_{n} x \rightarrow x$ for all $x \in \mathscr{H}$ and $n \rightarrow \infty$. Assume that $A \in \mathfrak{B}(\mathscr{H})$ is invertible. Consider

$$
P_{n} A P_{n} x=P_{n} y
$$

and solve for $x_{n}=\left(P_{n} A P_{n}\right)^{-1} P_{n} y$. This is the classical projection method [45]. The projection method for $A$ is said to converge, if for all $y \in \mathscr{H}$ there exists a unique solution $x_{n}$ to (30) with $x_{n} \rightarrow A^{-1} y$. This is the case [45] if and only if the matrices $A_{n}=P_{n} A P_{n}$ have uniformly bounded inverses, i.e. $\sup _{n \geq N}\left\|A_{n}^{-1}\right\|<\infty$. In particular, if $\|I-A\|<1$, then the method converges. The special case when $P_{n}$ is the orthogonal projection on the first $n$ coordinates in $\ell^{2}$ is called the finite section method. In numerical analysis, this approximation scheme is often called the Galerkin scheme.

If $|\langle A x, x\rangle| \geq c \cdot\|x\|^{2}$, then $A$ is invertible, $\left\|A^{-1}\right\| \leq c^{-1}$ and it is easy to see that the projection method converges.

A convergence analysis of the finite section method in weighted $\ell^{p}$-spaces is carried out in [58]. The methods are closely related to the methods used for the analysis of localized frames.

The projection method can be combined with frames in several ways:

- The "naive approach": assume that $K=\mathbb{Z}$ and choose $M_{N k, l}=\mathscr{M}_{(\Phi, \Phi)}(O)_{k, l}$ for $|k|,|l| \leq N$. This corresponds to a finite section method.

- Subspace selection: Choose a sequence $K_{N}$ of finite subsets of $K$ with the following properties:

(i) $K_{i} \subseteq K_{j}$ for $i \leq j$ and $\bigcup_{i=1}^{\infty} K_{i}=K$.

(ii) The space $V_{N}:=\operatorname{span}\left\{\psi_{k} \mid k \in K_{N}\right\}$ has dimension $N$.

For a frame $\Psi$ it may happen that $\operatorname{dim} V_{N}<\operatorname{card}\left(K_{N}\right)$, but the set $\Psi^{(N)}:=\left\{\psi_{k} \mid k \in\right.$ $\left.K_{N}\right\}$ is always a frame for $V_{N}$. (This need not be the case when $\operatorname{dim} V_{N}=\infty$ ). For the numerical treatment the condition numbers of the transforms, i.e. the quotients of the frame bounds, has to be controlled. Therefore we consider $\Psi^{(N)}=\left\{\psi_{k} \mid k \in K_{N}\right\}$ being a frame for $V_{N}$ with bounds $C, D$ independent of $N$. This is called a subframe in [60]. We denote the canonical dual on $V_{N}$ by $\widetilde{\Psi}^{(N)}:=$ $\left\{\tilde{\psi}_{k}^{(N)}\right\}$. Then use the projection $P_{N} f=\sum_{k \in K_{N}}\left\langle f, \psi_{k}\right\rangle \tilde{\psi}_{k}^{(N)}=\sum_{k \in K_{N}}\left\langle f, \tilde{\psi}_{k}^{(N)}\right\rangle \psi_{k}$ and solve $(30)$. Since $\operatorname{ran}\left(\mathscr{M}^{(\Phi, \Phi)}(O)\right) \subseteq \operatorname{ran}\left(C_{\Phi}\right)$, this set-up leads exactly to the formulation in 29 . 
In concrete applications it is a non-trivial problem to find index sets such that the approximation method converges and at the same time is numerically efficient. For wavelet frames this problem can be tackled with a multi-resolution approach with a basis property on each scale, see, e.g., [60].

We note that the matrix $M_{N}$ cannot have full rank whenever the frame $\left\{\psi_{k} \mid k \in K_{N}\right\}$ is redundant for $V_{N}$. By Lemma 11 the equation (29) still has a unique solution, although the matrix is not invertible. For the efficient solution of 29), even for frames, one can apply Krylov subspace methods, such as the conjugate gradient method [60]. Other possible methods include versions of Richardson iterations [27] or steepest descent methods [28].

Acknowledgements The first author was in part supported by the START project FLAME Y551N13 of the Austrian Science Fund (FWF) and the DACH project BIOTOP I-1018-N25 of Austrian Science Fund (FWF). The second author acknowledges the support of the FWF-project P 26273N25. P.B. wishes to thank NuHAG for the hospitality as well as the availability of its webpage. He also thanks Dominik Bayer, Gilles Chardon, Stephan Dahlke, Helmut Harbrecht, Wolfgang Kreuzer, Michael Speckbacher and Diana Stoeva for related interesting discussions.

\section{References}

1. S. T. Ali, J.-P. Antoine, and J.-P. Gazeau. Coherent States, Wavelets and Their Generalization. Graduate Texts in Contemporary Physics. Springer New York, 2000.

2. R. M. Balan. Equivalence relations and distances between Hilbert frames. Proc. Amer. Math. Soc., 127(8):2353-2366, 1999.

3. R. M. Balan, P. G. Casazza, C. Heil, and Z. Landau. Density, overcompleteness, and localization of frames I: Theory. J. Fourier Anal. Appl., 12(2):105-143, 2006.

4. P. Balazs. Frames and finite dimensionality: Frame transformation, classification and algorithms. Appl. Math. Sci., 2(41-44):2131-2144, 2008.

5. P. Balazs. Matrix-representation of operators using frames. Sampl. Theory Signal Image Process, 7(1):39-54, Jan. 2008.

6. P. Balazs and G. Rieckh. Redundant representation of operators. preprint.

7. P. Balazs and G. Rieckh. Oversampling operators: Frame representation of operators. Analele Universitatii "Eftimie Murgu", 18(2):107-114, 2011.

8. P. Balazs, D. Stoeva, and J.-P. Antoine. Classification of general sequences by frame-related operators. Sampl. Theory Signal Image Process, 10(2):151-170, 2011.

9. S. Brenner and L. Scott. The Mathematical Theory of Finite Element Methods. Springer New York, 2nd edition, 2002.

10. P. Casazza. The art of frame theory. Taiwanese J. Math., 4(2):129-202, 2000.

11. P. Casazza, O. Christensen, and N. J. Kalton. Frames of translates. Collectanea Mathematica, 1:p. 35-54, 2001.

12. P. Casazza, O. Christensen, and D. T. Stoeva. Frame expansions in separable Banach spaces. J. Math. Anal. Appl., 307(2):710 - 723, 2005.

13. P. G. Casazza and J. C. Tremain. The Kadison-Singer problem in mathematics and engineering. Proc. Natl. Acad. Sci. USA, 103(7):2032-2039, 2006.

14. G. Chen, Y. Wei and Y. Xue. The generalized condition numbers of bounded linear operators in Banach spaces J.Aust. Math. Soc., 76:281-29, 2004

15. O. Christensen. Frames and pseudo-inverses. J. Math. Anal. Appl, 195(2):401-414, 1995. 
16. O. Christensen. An Introduction to Frames and Riesz Bases. Birkhäuser, 2003.

17. O. Christensen and D. Stoeva. p-frames in separable Banach spaces. Adv. Comput. Math., 18(2-4):117-126, 2003.

18. J. B. Conway. A Course in Functional Analysis. Graduate Texts in Mathematics. Springer New York, 2. edition, 1990.

19. E. Cordero, K. Gröchenig, F. Nicola, and L. Rodino. Generalized metaplectic operators and the Schrödinger equation with a potential in the Sjöstrand class. J. Math. Phys. 55, 081506 (2014)

20. E. Cordero, K. Gröchenig, F. Nicola, and L. Rodino. Wiener algebras of Fourier integral operators. J. Math. Pures Appl. (9), 99(2):219-233, 2013.

21. E. Cordero and K. Gröchenig. Localization of frames II. Appl. Comp. Harm. Anal., 17:29-47, 2004.

22. E. Cordero, F. Nicola, and L. Rodino. Sparsity of Gabor representation of Schrödinger propagators. Appl. Comput. Harmon. Anal., 26(3):357-370, 2009.

23. E. Cordero, F. Nicola, and L. Rodino. Exponentially sparse representations of Fourier integral operators. Rev. Mat. Iberoam., 31:461-476, 2015.

24. E. Cordero, F. Nicola, and L. Rodino. Gabor representations of evolution operators. Trans. Amer. Math. Soc., 367(11):7639-7663, 2015.

25. L. Crone. A characterization of matrix operator on $l^{2}$. Math. Z., 123:315-317, 1971.

26. S. Dahlke, M. Fornasier, and K. Gröchenig. Optimal adaptive computations in the Jaffard algebra and localized frames. J. Approx. Theory, 162:153-185, 2010.

27. S. Dahlke, M. Fornasier, and T. Raasch. Adaptive Frame Methods for Elliptic Operator Equations. Adv. Comput. Math., 27(1):27-63, 2007.

28. S. Dahlke, T. Raasch, M. Werner, M. Fornasier, and R. Stevenson. Adaptive frame methods for elliptic operator equations: The steepest descent approach. IMA J. Numer. Anal., 27(4):717740, 2007.

29. W. Dahmen and R. Schneider. Composite wavelet basis for operator equations. Math. Comp., 68:1533-1567, 1999.

30. I. Daubechies. Ten Lectures On Wavelets. CBMS-NSF Regional Conference Series in Applied Mathematics. SIAM Philadelphia, 1992.

31. M. De Gosson, K. Gröchenig, and J. L. Romero. Stability of Gabor frames under small time Hamiltonian evolutions. Lett. Math. Phys., 106(6):799-809, 2016.

32. H. G. Feichtinger. Modulation Spaces: Looking Back and Ahead. Sampl. Theory Signal Image Process., 5(2):109-140, 2006.

33. H. G. Feichtinger, H. Führ, and I. Z. Pesenson. Geometric space-frequency analysis on manifolds. J. Fourier Anal. Appl., online:1-62, 2016.

34. H. G. Feichtinger and K. Gröchenig. Banach spaces related to integrable group representations and their atomic decompositions, I. J. Funct. Anal., 86(2):307-340, 1989.

35. H. G. Feichtinger and K. Gröchenig. Banach spaces related to integrable group representations and their atomic decompositions, II. Monatsh. Math., 108(2-3):129-148, 1989.

36. H. G. Feichtinger and T. Strohmer. Gabor Analysis and Algorithms - Theory and Applications. Birkhäuser Boston, 1998.

37. P. Flandrin. Time-Frequency/Time-Scale Analysis. Academic Press, San Diego, 1999.

38. M. Fornasier and K. Gröchenig. Intrinsic localization of frames. Constr. Approx., 22(3):395415, 2005.

39. M. Fornasier and H. Rauhut. Continuous frames, function spaces, and the discretization problem. J. Fourier Anal. Appl., 11(3):245-287, 2005.

40. M. Frazier and B. Jawerth. A discrete transform and decompositions of distribution spaces. $J$. Functional Anal., 93(1):34-170, 1990.

41. F. Futamura. Localizable operators and the construction of localized frames. Proc. Amer. Math. Soc., 137(12):4187-4197, 2009.

42. R. C. G. Beylkin and V. . Rokhlin. Fast wavelet transforms and numerical algorithms i. Comm. Pure Appl. Math., 44(2):141-183, 1991.

43. L. Gaul, M. Kögler, and M. Wagner. Boundary Element Methods for Engineers and Scientists. Springer, 2003. 
44. D. Geller and I. Pesenson. Band-limited localized Parseval frames and Besov spaces on compact homogeneous manifolds. J. Geom. Anal., 21(2):334-371, 2011.

45. I. Gohberg, S. Goldberg, and M. A. Kaashoek. Basic Classes of Linear Operators. Birkhäuser, 2003.

46. K. Gröchenig. Describing functions: atomic decompositions versus frames. Monatsh. Math., 112(3):1-41, 1991.

47. K. Gröchenig. Foundations of Time-Frequency Analysis. Birkhäuser Boston, 2001.

48. K. Gröchenig. Localization of frames, pages 875-882. IOP Publishing, 2003.

49. K. Gröchenig. Localized frames are finite unions of Riesz sequences. Adv. Comput. Math., 18(2-4):149-157, 2003.

50. K. Gröchenig. Localization of Frames, Banach Frames, and the Invertibility of the Frame Operator. J. Fourier Anal. Appl., 10(2):105-132, 2004.

51. K. Gröchenig. Time-frequency analysis of Sjöstrand's class. Rev. Mat. Iberoam., 22(2):703724, 2006.

52. K. Gröchenig. Gabor frames without inequalities. Int. Math. Res. Not. IMRN, 2007(23):ID rnm111, 21, 2007.

53. K. Gröchenig. Wiener's lemma: Theme and variations. An introduction to spectral invariance and its applications., in "Four Short Courses on Harmonic Analysis. Wavelets, Frames, TimeFrequency Methods, and Applications to Signal and Image Analysis". With B. Forster, P. Massopust, O. Christensen, D. Labate, P. Vandergheynst, G. Weiss, Y. Wiaux. Chapter 5, pages 175 - 234. Applied and Numerical Harmonic Analysis. Birkhäuser, 2010.

54. K. Gröchenig and A. Klotz. Noncommutative approximation: Inverse-closed subalgebras and off-diagonal decay of matrices. Constr. Approx., 32:429 - 466, 2010.

55. K. Gröchenig and M. Leinert. Symmetry and inverse-closedness of matrix algebras and symbolic calculus for infinite matrices. Trans. Amer. Math. Soc., 358:2695-2711, 2006.

56. K. Gröchenig, J. Ortega Cerdà, and J. L. Romero. Deformation of Gabor systems. Adv. Math., 277(4):388-425, 2015.

57. K. Gröchenig and Z. Rzeszotnik. Banach algebras of pseudodifferential operators and their almost diagonalization. Ann. Inst. Fourier (Grenoble), 58(7):2279-2314, 2008.

58. K. Gröchenig, Z. Rzeszotnik, and T. Strohmer. Convergence analysis of the finite section method and Banach algebras of matrices. Integr. Equ. Oper. Theory, 67(2):183-202, 2010

59. H. Harbrecht and R. Schneider. Wavelet Galerkin schemes for boundary integral equations. Implementation and quadrature. SIAM J. Sci. Comput., 27(4):1347-1370, 2006.

60. H. Harbrecht, R. Schneider, and C. Schwab. Multilevel frames for sparse tensor product spaces. Numer. Math., 110(2):199-220, 2008.

61. C. Kasess, W. Kreuzer, and H. Waubke. Deriving correction functions to model the efficiency of noise barriers with complex shapes using boundary element simulations. Applied Acoustics, 102:88-99, 2016.

62. G. Köthe. Toplogische lineare Räume. Die Grundlehren der mathematische Wissenschaften. Springer-Verlag Berlin, 1960.

63. W. Kozek. Adaption of Weyl-Heisenberg frames to underspread environments, chapter 10 , pages 323-352. In [36], 1998.

64. W. Kreuzer, P. Majdak, and Z. Chen. Fast multipole boundary element method to calculate head-related transfer functions for a wide frequency range. J. Acoust .Soc. Am., 126(3):12801290, 2009.

65. N. Lindholm. Sampling in weighted $L^{p}$ spaces of entire functions in $\mathbb{C}^{n}$ and estimates of the Bergman kernel. J. Funct. Anal., 182(2):390-426, 2001.

66. D. Luenberger, Linear And Nonlinear Programming. Addison-Wesley, Reading, 1984

67. I. J. Maddox. Infinite matrices of operators. Lecture Notes in Mathematics. Springer, Berlin, 1980.

68. A. Marcus, D. Spielman, and N. Srivastava. Interlacing families II: Mixed characteristic polynomials and the Kadison-Singer problem. Ann. of Math., 182(1):Pages 327-350 from Volume 182 (2015), Issue 1, 2015.

69. Y. Meyer. Ondelettes et Operateurs I. Hermann, Paris, 1990. 
70. Y. Meyer. Ondelettes et Operateurs II: Operateurs de Calderon-Zygmund. (Wavelets and Operators II: Calderon-Zygmund Operators). Hermann, Editeurs des Sciences et des Arts, 1990.

71. I. Pesenson. Sampling, splines and frames on compact manifolds. GEM Int. J. Geomath., 6(1):43-81, 2015.

72. G. Rieckh, W. Kreuzer, H. Waubke, and P. Balazs. A 2.5d-fourier-bem-model for vibrations in a tunnel running through layered anisotropic soil. Engineering Analysis with Boundary Elements, 36:960-967, 2012.

73. S. Sauter and C. Schwab. Boundary Element Methods. Springer Series in Computational Mathematics. Springer Berlin Heidelberg, 2010.

74. M. Speckbacher, D. Bayer, S. Dahlke, and P. Balazs. The $\alpha$-modulation transform: Admissibility, coorbit theory and frames of compactly supported functions. arXiv:1408.4971 2014.

75. R. Stevenson. Adaptive solution of operator equations using wavelet frames. SIAM J. Numer. Anal., 41(3):1074-1100, 2003.

76. Q. Sun.

Wiener's lemma for infinite matrices. Trans. Amer. Math. Soc., 359(7):3099-3123 (electronic), 2007

77. Q. Sun. Local reconstruction for sampling in shift-invariant spaces. Adv. Comput. Math., 32(3):335-352, 2010.

78. T. Ullrich and H. Rauhut. Generalized coorbit space theory and inhomogeneous function spaces of Besov-Lizorkin-Triebel type. J. Funct. Anal., 11:3299-3362, 2011.

79. H. Ziegelwanger, P. Majdak, and W. Kreuzer. Numerical calculation of head-related transfer functions and sound localization: Microphone model and mesh discretization. J. Acoustical Soc. Amer., 138(1):208-222, 2015. 